\title{
Decreased Temporomandibular Joint Range of Motion in a Model of Early Osteoarthritis in the Rabbit
}

\author{
Sarah E. Henderson Ph.D. ${ }^{1}$, Mauro A. Tudares M.S. ${ }^{2}$, Scott Tashman Ph.D. ${ }^{3}$, and Alejandro J. \\ Almarza Ph.D. ${ }^{1,2,4 *}$ \\ ${ }^{1}$ Department of Bioengineering, University of Pittsburgh, Pittsburgh, PA \\ ${ }^{2}$ Department of Oral Biology, University of Pittsburgh, Pittsburgh, PA \\ ${ }^{3}$ Department of Orthopaedic Surgery, University of Pittsburgh, Pittsburgh, PA \\ ${ }^{4}$ McGowan Institute of Regenerative Medicine, University of Pittsburgh, Pittsburgh, PA \\ *Corresponding Author \\ Department of Oral Biology, University of Pittsburgh, \\ 566 Salk Hall, 3501 Terrace Street, Pittsburgh, PA, 15261, USA \\ Telephone: 412-648-8500 \\ Fax: 412-624-6685 \\ Email: aja19@pitt.edu
}




\section{ABSTRACT}

Purpose: Analysis of mandibular biomechanics may help with understanding the mechanisms of temporomandibular joint (TMJ) disorders (TMJDs), such as osteoarthritis (TMJ-OA), by investigating the effects of injury or disease on TMJ movement. The objective of this study was to determine the functional kinematic implications of mild TMJ-OA degeneration caused by altered occlusion from unilateral splints in the rabbit.

Methods: Altered occlusion of the TMJ was mechanically induced in rabbits via a unilateral molar dental splint $(n=3)$. TMJ motion was assessed through three-dimensional skeletal kinematics twice, once before and once after 6 weeks of splint placement with the splints removed allowing three days of recovery. The relative motion of the condyle to the fossa was tracked, as well as the distance between the incisors.

Results: An overall decrease in the range of joint movement was observed at the incisors and in the joint space between the condyle and fossa. The incisor movement decreased from $7.0 \pm 0.5$ $\mathrm{mm}$ to $6.2 \pm 0.5 \mathrm{~mm}$ right-left, from $5.5 \pm 2.2 \mathrm{~mm}$ to $4.6 \pm 0.8 \mathrm{~mm}$ anterior-posterior, and from $13.3 \pm 1.8 \mathrm{~mm}$ to $11.6 \pm 1.4 \mathrm{~mm}$ superior-inferior $(\mathrm{p}<0.05)$. The total magnitude of the maximum distance between the points on the condyle and fossa decreased from $3.6 \pm 0.8 \mathrm{~mm}$ to $3.1 \pm 0.6 \mathrm{~mm}$ for the working condyle and from $2.8 \pm 0.4 \mathrm{~mm}$ to $2.5 \pm 0.4 \mathrm{~mm}$ for the balancing condyle $(\mathrm{p}<0.05)$. The largest decreases were seen in the anterior-posterior direction for both condyles. Conclusions: Determining the changes in condylar movement may lead to a better understanding of early predictors in the development of TMJ-OA, and determining when symptoms become a chronic, irreversible problem. 


\section{INTRODUCTION}

For patients suffering from pain associated with temporomandibular joint (TMJ) disorders (TMJDs), normal life activities such as eating, talking, and even sleeping may be drastically impaired. TMJDs are multifactorial and complex [1]. Signs and symptoms of TMJDs include joint pain, limited mouth opening, deviation of the jaw, clicking, locking, dislocation, and pain in the masticatory muscles during jaw movement [2]. While a heterogeneous syndrome that may involve the joint as well as the muscles of mastication, osteoarthritis (OA) is present in up to $15 \%$ of those suffering from TMJD [3], and pain is the primary complaint associated with the loss of function $[1,4,5]$. TMJ-OA is the pathological process of joint degeneration including irreparable abrasion of articular cartilage and thickening and remodeling of underlying bone [6]. A number of potential causes of TMJ-OA have been implicated, including trauma and parafunctional habits such as teeth clenching and grinding.

We hypothesize that altered joint movement/travel path (kinematics), caused by trauma or parafunctional habits, are one of the underlying mechanisms of progressive TMJ-OA. Establishing the link between altered TMJ kinematics and joint degeneration may have an immediate clinical impact. Understanding this relationship may provide guidance to surgeons on the window in which interventions to normalize malocclusion associated with trauma may be most successful. This knowledge may support the use of TMJ kinematic analysis as a diagnostic tool with which to identify the basis for TMJ pain in at least a subpopulation of TMJD patients. They may also suggest that strategies to normalize altered TMJ kinematics may be effective for the treatment of TMJ pain in these patients.

Establishing a link between kinematics and TMJ-OA has been hindered by the dearth of data linking the changes observed in preclinical animal models to the development and 
manifestation of the pathology of the human state. Of ongoing debate is the question of whether TMJ disc displacement precedes degeneration of the condylar cartilage. As a potential explanation for the relatively limited efficacy of interventions focused on the TMJ disc, results from our rabbit TMJ-OA model have shown that condylar cartilage remodeling (stiffening and loss of the subchondral layer) occurs with a disc in its proper anatomical position [7]. As the condylar cartilage resembles early stages of OA, it is not known whether TMJ kinematics were altered. If the joint kinematics do not return to normal, they can be a primary driver for further joint degeneration and end stage OA.

The objective of this study was to determine the functional kinematic implications of the TMJ with mild OA degeneration. In our model, OA is caused by altered occlusion from unilateral splints placed for six weeks. It was hypothesized that kinematic analysis would identify an overall decrease in joint motion after six weeks of splinting.

\section{METHODS}

\section{Animal Model}

Skeletally mature, female, New Zealand White rabbits approximately 1 year in age weighing between 5-7 kg were purchased from Charles River Laboratories International, Inc. (Wilmington, MA). All rabbits were examined by a veterinarian prior to use in the study and were found to be in good health. All animal procedures were approved by the Institutional Animal Care and Use Committee at the University of Pittsburgh and in accordance with the National Institutes of Health guidelines for the use of laboratory animals. 
Unilateral dental splints were chosen as the method for altering the occlusion because the splints could be removed at a later point in time and the joint space was not penetrated [8-10]. For three separate procedures, impressions, splint placement, and splint removal, all rabbits were sedated with ketamine and xylazine, and a surgical plane of anesthesia was maintained with isoflurane. Impressions were taken of the upper and lower right molars of the rabbits to create a unique mold for casting the splints as crowns made from non-precious metals (Figure 1). The thickness of each casted splint was approximately $1 \mathrm{~mm}$. During a second procedure, the right maxillary and mandibular molar arches were cleaned and primed, and then the splints were attached to the respective molars with dental cement. Splint placement was verified after 1 week. Splints were removed at 6 weeks, three days prior to the final kinematic data collection. Kinematic data was collected twice, once before impressions and splint placement, and once 6 weeks after splint placement, with the splints removed for three days on three rabbits $(n=3)$. After the second kinematic data collection, the rabbits were euthanized and the whole head was frozen and saved for micro-computed tomography (CT).

\section{Kinematics}

While biaxial x-ray systems have been used to assess the impact of injury on the structure-function relationship of knees and shoulders in humans [11-13], it was necessary to determine whether this system could be used for this purpose in the rabbit. Using high resolution micro-CT, we were able to demonstrate the feasibility of this system for the analysis of rabbit TMJ movements during chewing [14]. We determined that rabbit TMJ kinematic were measureable and repeatable, including maximal distances and movement paths for both the incisors and the condyle-fossa relationship. 
As per these published methods [14], TMJ kinematics were assessed using a unique highspeed stereo-radiographic system consisting of two sets of $110 \mathrm{~kW}$ pulsed X-ray generators (CPX 3100CV; EMD Technologies, Quebec, Canada), $40 \mathrm{~cm}$ image intensifiers (Thales, Neuilly-sur-Seine, France) using a $20 \mathrm{~cm}$ field of view, and high-speed 4 Megapixel digital video cameras (Phantom v10, Vision Research, Wayne, New Jersey, USA). The rabbits were placed in a radiolucent ventilated cage (Figure $2 \mathrm{~A}$ ) and fed small pieces of dried fruit. Radiographic images were collected at 170 frames/s for $1 \mathrm{~s}$ with $1 \mathrm{~ms}$ exposures at 4 Megapixel image resolution (Figure 2B). At least three data acquisitions per rabbit were collected. Three rabbits were tested before splint placement and after 6 weeks of splint placement with the splints removed three days prior to the second data collection.

The markerless, model-based technique used for determining 3D kinematics has been extensively described, validated, and tested [11, 13-16], including testing of the rabbit TMJ [14]. Briefly, a 3D model of each bone to be tracked was derived from a CT scan (as described below) (Figure 2C). A virtual model of the biplane $\mathrm{x}$-ray system was created for generating a pair of digitally reconstructed radiographs (DRR's) via ray-traced projection through the CT bone model. The bone model was automatically repositioned within the virtual model until a best match was achieved between the simulated DRR's and the actual radiographic image pair (Figure 2D). This technique determined the 3D positions and orientations of the mandible and the skull for each motion frame. After euthanasia, the entire head, including the mandible and skull, was scanned at high resolution (105 $\mu \mathrm{m}$ isotropic voxels) using a micro-CT system (Inveon micro-CT system by Siemens located at the Rangos Research Center Animal Imaging Core, Children's Hospital of Pittsburgh of UPMC). The mandible and skull bones (with teeth) 
were segmented from each other and from soft tissue and reconstructed into both surface and volumetric models using Mimics software (Materialise, Inc. Leuven, Belgium) (Figure 2C).

Anatomical coordinate systems were set up similar to the systems used by Brainerd et al. [17] and in our previous study [14] to determine the joint translations and rotations. The transverse ( $\mathrm{x}$ ) axis was aligned through the center of the condyles, the longitudinal (y) axis was parallel to a plane through the occlusal plane, and the vertical (z) axis was the cross product of $\mathrm{x}$ and y (Figure 3A).

The relative motion of each condyle with respect to the fossa was tracked (Figure 3B and 3C). The distance between a fixed point on the condyle and a fixed point on the fossa was measured throughout the chewing cycles (Figure 2Ei). Points were placed at the superior most point of the condyle and in the convex region of the fossa. The relative motions of the centers of the two points were determined in all 3 anatomic planes. The measurement points were placed in the same location on the condyle and fossa for pre and post splint data collection for each rabbit. The pre and post splint motions were analyzed in each direction, as well as in the total magnitude of the vector (Figure 2Eii). The movement of the mandible was somewhat elliptical in the coronal plane and always started to the same side during an acquisition. As established in the literature, the side in which the mandible first moves is the working side, while the other is called the balancing side [18]. The condyles were analyzed by working and balancing condyles as previously established in the literature $[14,18]$. Of the three rabbits, one rabbit consistently had the left condyle as the working condyle, one rabbit had the right condyle consistently as the working condyle, and the third rabbit the working condyle switched from the right condyle pre splint to left condyle post splint. Again, the splint was placed on the right molars. 
The relative motion near the incisors was measured as well. Since, the incisor teeth were observed to grow and change shape from the treatment they were not directly included in the analysis. To be consistent with the changing incisors from pre and post splint, the incisor markers were instead placed on the skull and mandible at the center of the incisors at the interface where the teeth met the bone on the posterior side (Figure 3D).

Based on our previous study [14], some of the rotation-translation relationships were shown to be linear and repeatable, so the slopes of these relationships were also compared. The slope of the movement of the condyles in the superior-inferior direction with respect to the transverse rotation during the first $50 \%$ of closing was determined for both the working and balancing condyles. The slopes of the paths from the incisal point in the superior-inferior direction with respect to the transverse rotation and the incisal point in the anterior-posterior direction with respect to the vertical rotation were also determined. Only one acquisition was analyzed for each rabbit pre and post splint, if the acquisition had at least three complete chewing cycles. All kinematic data was reported as an average \pm standard deviation. Data was tested for normality with an Anderson Darling Test for Normality and found to be normal. A repeated measures ANOVA with Tukey's post-hoc test $(\mathrm{p}<0.05)$ was used to assess the differences for all kinematics values before and after splinting. 


\section{RESULTS}

\section{Kinematics}

Overall, the altered occlusion from the splint caused the range of motion of the condyles and the teeth to decrease. The distances the condyles traveled on both the working and balancing sides significantly decreased after splinting (Table 1). The total magnitude of the maximum distance between the points on the condyle and fossa decreased by $15.0 \%$ for the working condyle and by $8.9 \%$ for the balancing condyle $(\mathrm{p}<0.05)$. Figure $4 \mathrm{~A}$ and $\mathrm{B}$ shows the total magnitude of the condyle-fossa distance over time and the decrease in distance traveled from the splint. Breaking the movement into the vector components, both the anterior-posterior displacement and the superior-inferior displacement significantly decreased $(\mathrm{p}<0.05)$. The anterior-posterior displacement decreased by $20.5 \%$ and the superior-inferior by $8.9 \%$ for the working condyle and for the balancing condyle the displacements decreased by 14.9 and $13.6 \%$, respectively (Table 1). Figure 4C and D show the superior-inferior motion with respect to the anterior-posterior motion, for the working and balancing condyles, pre and post splint. The right-left movement of the condyles was minimal and thus, not reported.

The paths of the condyles were analyzed by determining slope of the superior-inferior distance with respect to the transverse rotation during the first $50 \%$ of the closing cycle. The slopes for the working condyle significantly increased by $13.7 \%$ after splinting $(\mathrm{p}<0.05)($ Table 1). The shapes of the curves are shown in Figure $4 \mathrm{E}$ and $\mathrm{F}$ for the working and balancing condyles, respectively.

The distances the incisors traveled also significantly decreased after splinting $(\mathrm{p}<0.05)$ (Table 2). The right-left movement decreased by $11.2 \%$. The anterior-posterior movement decreased by $16.1 \%$. The superior-inferior movement decreased by $13.0 \%$. The shapes of the 
curves are shown in Figure 5A and B. Figure 5C shows the change in movement from pre to post-splint in the coronal plane.

\section{DISCUSSION}

An overall decrease in the range of joint motion at both the condyles and the incisors was observed after placement of dental splints for six weeks. The persistent change in kinematics is likely due to the abnormal occlusion and the degeneration of the condylar fibrocartilage observed in our previous studies [7, 19]. The kinematics of the rabbit TMJ at the condyles and the incisors were easily measured with our non-invasive system before and after splint placement to determine changes in the joint movement. The decreased range in incisal movement post splint was comparable to a previous TMJ disc injury study [20]; however, the specific values could not be compared due to different measurement techniques. The pre splint control incisal data was also consistent with our previous study and with other normal rabbit kinematic studies $[14,18$, $20,21]$.

Determining the changes in condylar movement may lead to a better understanding of early movement predictors in the development of TMJDs. This knowledge of dynamic TMJ function through 3D skeletal kinematics is essential for investigating the effects of injury or disease in the TMJ. The altered occlusion model used in this study has been shown to produce condylar fibrocartilage degeneration that would likely not be detected by conventional MRI [7, 19]. Understanding the changes in joint movement that occur from various types of TMJDs could allow for earlier diagnosis and treatment, for example, if a certain signature of changes in movement are observed earlier before onset of severe joint degeneration. As such, our study 
suggests that decreases in $20 \%$ anterior-posterior displacement of the working condyle might be indicative of condylar degeneration and early stage TMJ-OA.

A limitation of this study was that only one microCT scan could be obtained per rabbit head after the final time point. Ideally a microCT scan would be collected for each time point that kinematic data was collected, this would also allow for analysis of the effect of the splints on the bony structure. However, due to the size limitations of the scanner, the heads could only be scanned after euthanasia and decapitation. With only one scan, it was assumed that the bony structures of the joints were not affected by the treatment. Another assumption of this study was that the bony structure of the skull and mandible were rigid. However, it has been shown that the mandible can deform during joint motion [22, 23], which led to some minor errors in the DDR matching process. There were a few frames in each chew cycle where the mandible and fossa were barely touching along the edges of both of the condyles. Rabbits' teeth are also constantly erupting, so the teeth were ignored throughout the analysis. In future studies with longer time points the teeth may need to be filed down to their original height. The possible changes in muscle performance were also not considered in this study. Even with these limitations, this study provided an important initial understanding of what can change in the TMJ motion from inducing TMJ-OA with altered occlusion.

The splint model used in this study was advantageous because it allowed for removal of the splints, and the joint space was not penetrated. Future work will need to look at various lengths of time between splint placement and removal and allow time for recovery to understand if and when the change in joint movement has the potential to correct itself and the joint to heal or when the TMJ-OA becomes chronic and permanent. The potential exists with this type of TMJD animal model to combine the kinematic analysis with other assessments such as pain 
measurements and mechanical assessments of the TMJ tissues, which will allow for multidisciplinary studies of various types of TMJDs. The combination of assessment methods will allow for a fuller understanding of the TMJ disease progression in this and other animal models.

\section{ACKNOWLEDGEMENTS}

We would like acknowledge Robert Mortimer for help with the rabbit kinematic data collection and funding from the National Science Foundation under grant number 0812348, from the National Institutes of Health under grant number T32 EB003392, the University of Pittsburgh Research Fund, and the University of Pittsburgh School of Dental Medicine.

\section{Conflict of Interest Statement}

The authors have no conflicts of interest to disclose. 


\section{REFERENCES}

1. Gray RJM, Davies SJ, Quayle AA: Temporomandibular Disorders: A Clinical Approach. London, British Dental Association, 1995

2. Solberg WK: Epidemiology, incidence and prevalence of temporomandibular disorders: a review. In Laskin D, Greenfield W, Gale E, Rugh J, Neft D, Alling C, Dyer WA (eds), Diagnosis and Management of Temporomandibular Disorders, (ed. Chicago, IL, American Dental Association, 1983, p 30

3. Mejersjo C, Hollender L: Radiography of the temporomandibular joint in female patients with TMJ pain or dysfunction. A seven year follow-up. Acta Radiologica - Series Diagnosis 25:169, 1984

4. Ware WH: Clinical presentation. In Helms CA, Katzberg RW, Dolwick MF (eds), Internal Derangements of the Temporomandibular Joint, (ed. San Francisco, CA, Radiology Research and Education Foundation, 1983, p 15

5. Jagger RG, Bates JF, Kopp S: Temporomandibular Joint Dysfunction: Essentials. Oxford, Butterworth-Heinemann Ltd., 1994

6. Zarb GA, Carlsson GE: Temporomandibular Disorders: Osteoarthritis. Journal of Orofacial Pain 13:295, 1999

7. Henderson SE, Lowe JR, Tudares MA, Gold MS, Almarza AJ: Temporomandibular joint fibrocartilage degeneration from unilateral dental splints. Archives of Oral Biology 60:1, 2015

8. Shaw RM, Molyneux GS: The effects of induced dental malocclusion on the fibrocartilage disc of the adult rabbit temporomandibular joint. Archives of Oral Biology 38:415, 1993 
9. Sergl HG, Farmand M: Experiments with unilateral bite planes in rabbits. Angle Orthodontist 45:108, 1975

10. Chaves K, Munerato MC, Ligocki A, Lauxen I, De Quadros OF: Microscopic Analysis of the Temporomandibular Joint in Rabbits (Oryctolagus cuniculus L.) Using An Occlusal Interference. Cranio 20:116, 2002

11. Bey MJ, Zauel R, Brock SK, Tashman S: Validation of a new model-based tracking technique for measuring three-dimensional, in vivo glenohumeral joint kinematics. Journal of Biomechanical Engineering 128:604, 2006

12. Bey MJ, Kline SK, Tashman S, Zauel R: Accuracy of biplane x-ray imaging combined with model-based tracking for measuring in-vivo patellofemoral joint motion. Journal of Orthopaedic Surgery and Research 3:38, 2008

13. Tashman S, Kolowich P, Collon D, Anderson K, Anderst WJ: Dynamic Function of the ACL-reconstructed Knee during Running. Clinical Orthopaedics and Related Research 454:66, 2007

14. Henderson SE, Desai R, Tashman S, Almarza AJ: Functional Analysis of the Rabbit Temporomandibular Joint Using Dynamic Biplane Imaging. Journal of Biomechanics 47:1360, 2014

15. Tashman S, Anderst WJ: In-vivo measurement of dynamic joint motion using high speed biplane radiography and CT: application to canine ACL deficiency. Journal of Biomechanical Engineering 125:238, 2003

16. Craig M, Bir C, Viano D, Tashman S: Biomechanical response of the human mandible to impacts of the chin. Journal of Biomechanics 41:2972, 2008 
17. Brainerd EL, Baier DB, Gatesy SM, Hedrick TL, Metzger KA, Gilbert SL, Crisco JJ: XRay reconstruction of moving morphology (XROMM): Precision, accuracy and applications in comparative biomechanics research. Journal of Experimental Zoology Part A: Ecological Genetics and Physiology 313 A:262, 2010

18. Morita T, Fujiwara T, Negoro T, Kurata C, Maruo H, Kurita K, Goto S, Hiraba K: Movement of the mandibular condyle and activity of the masseter and lateral pterygoid muscles during masticatory-like jaw movements induced by electrical stimulation of the cortical masticatory area of rabbits. Archives of Oral Biology 53:462, 2008

19. Henderson SE, Tudares MA, Gold MS, Almarza AJ: Analysis of Pain in the Rabbit Temporomandibular Joint after Unilateral Splint Placement. Journal of Oral and Facial Pain and Headache, 2014

20. Tominaga K, Yamada Y, Fukuda J: Changes in chewing pattern after surgically induced disc displacement in the rabbit temporomandibular joint. Journal of Oral and Maxillofacial Surgery 58:400, 2000

21. Huff KD, Asaka Y, Griffin AL, Berg WP, Seager MA, Berry SD: Differential mastication kinematics of the rabbit in response to food and water: Implications for conditioned movement. Integrative Physiological and Behavioral Science 39:16, 2004

22. Chen DC, Lai YL, Chi LY, Lee SY: Contributing factors of mandibular deformation during mouth opening. Journal of Dentistry 28:583, 2000

23. Al-Sukhun J, Helenius M, Lindqvist C, Kelleway J: Biomechanics of the Mandible Part I: Measurement of Mandibular Functional Deformation Using Custom-Fabricated Displacement Transducers. Journal of Oral and Maxillofacial Surgery 64:1015, 2006 
Table 1.

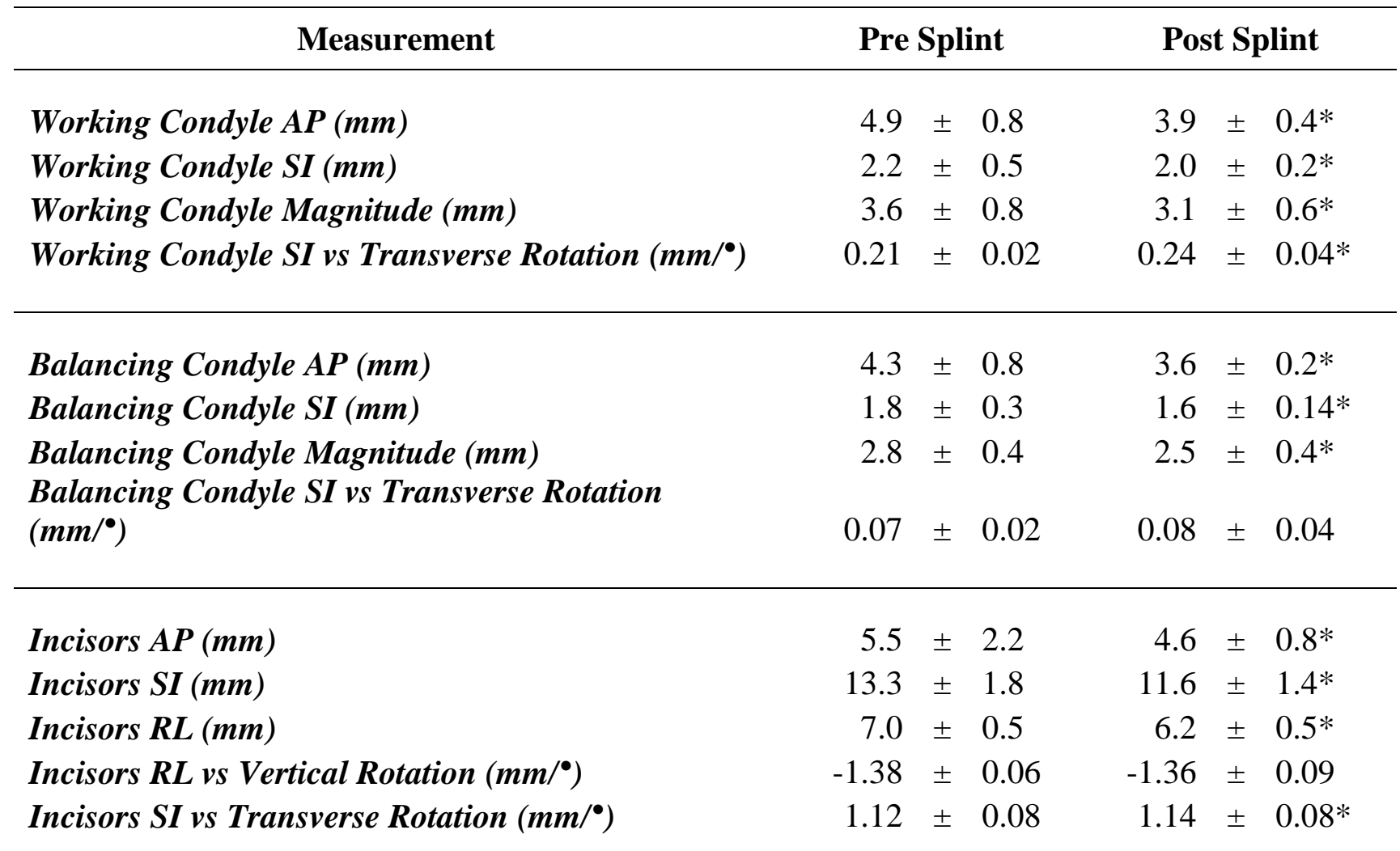

Figure and Table Captions

Figure 1. Unilateral molar bite raising splints. A) The splints were made by first taking an impression of the molars. B) A plaster mold was made from the impressions. C) The metal splints were cast as crowns on the molds, and the superior and inferior views are shown.

Figure 2. Overview of methods for kinematic data collection. A) High-speed biplane radiography was used for image collection. Rabbits were placed in a radiolucent box between two sets of x-ray generators and image intensifiers with high speed cameras. B) Sample radiographic image from the high resolution cameras at one frame in time. C) 3D bone model 
created from a microCT scan of the rabbit head. D) For 2D to 3D registration, the collected radiographic images (pink) are matched frame by frame with the virtual radiographic images (green) from the CT scan. One of the two combined images is shown for each the mandible (i) and the skull (ii). E) 3D distances can be measured. (i) Vector indicating the distance between the condylar point and the fossa point (the condyle is labeled with $\mathrm{C}$ and the fossa with F). (ii) Sample displacement curve. The picture in E is labeled by directions: A-anterior, P-posterior, Mmedial, L-lateral, S-superior, I-inferior.

Figure 3. Kinematic analysis tools were used to set up the measurements. A) The anatomical coordinate system for the mandible was set up with the transverse axis (x-red) aligned through the center of the condyles, the longitudinal axis (y-green) parallel to a plane through the occlusal plane (where the teeth meet), and the vertical axis (z-blue) was the cross product of the transverse and longitudinal axes. The skull coordinate system was set up in a similar manner (not shown). B) Condylar measurement point, anterior view of left condyle. C) Fossa measurement point, inferior view of left fossa. D) Mandible incisor measurement point, superior view. Pictures are labeled by directions: A-anterior, P-posterior, M-medial, L-lateral, Le-left, Rright, S-superior, I-inferior.

Figure 4. Condyle-fossa relationships pre and post splint. A) Working and B) Balancing side condylar kinematics showing the total condyle-fossa distance with respect to time, pre and post splinting. C) Working and D) Balancing side condylar kinematics showing the superior-inferior condyle-fossa distance with respect to the anterior-posterior condyle-fossa distance, pre and post splinting. E) Working and F) Balancing side condylar kinematics showing the superior-inferior 
condyle-fossa distance with respect to the transverse rotation, pre and post splinting. Representative curves from one rabbit shown.

Figure 5. Patterns of incisor movement pre and post splint. A) The change in distance of the mandible incisors from the skull incisors with relation to time pre splint and B) post splint. C) Relationship of the mandible incisors to the skull incisors in the coronal plane pre and post splint. Three chewing cycles are shown in each graph. Representative curves from one rabbit shown.

Table 1. Kinematics results for rabbits before and after splinting for 6 weeks, with the splint removed three days prior to testing. The data was separated into the working and balancing condyle and the incisors. AP: anterior-posterior movement. SI: superior-inferior movement. RL: right-left movement. The average and standard deviation values are presented. *Differences were significant when $\mathrm{p}<0.05$.

\section{Supplemental Video Information}

Videos illustrating the chewing pattern of a rabbit pre and post splint have been included as supplementary online information.

\section{Video Captions}

Video 1: Illustrates the chewing pattern of one rabbit before splinting, from an anterior view. It should be noted that the CT scans are from post splinting. 
Video 2: Illustrates the chewing pattern of the same rabbit in video 1 post splinting, with the splint removed for 3 days prior to data collection, from an anterior view.

Video 3: Illustrates the movement of the working condyle of the same rabbit pre splint, from a posterior and slightly superior view.

Video 4: Illustrates the movement of the working condyle of the same rabbit post splint, from a posterior and slightly superior view. 


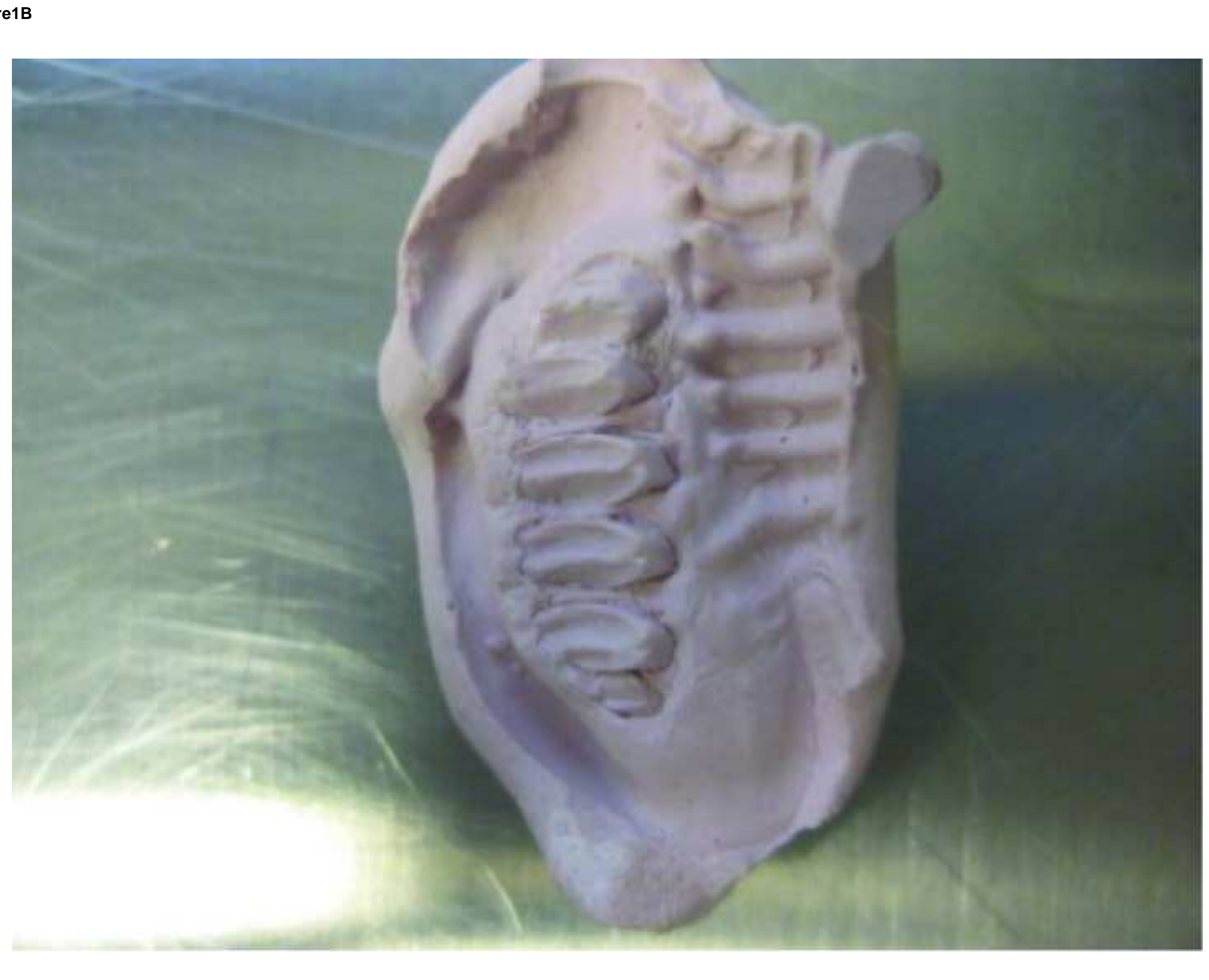

Figure1B
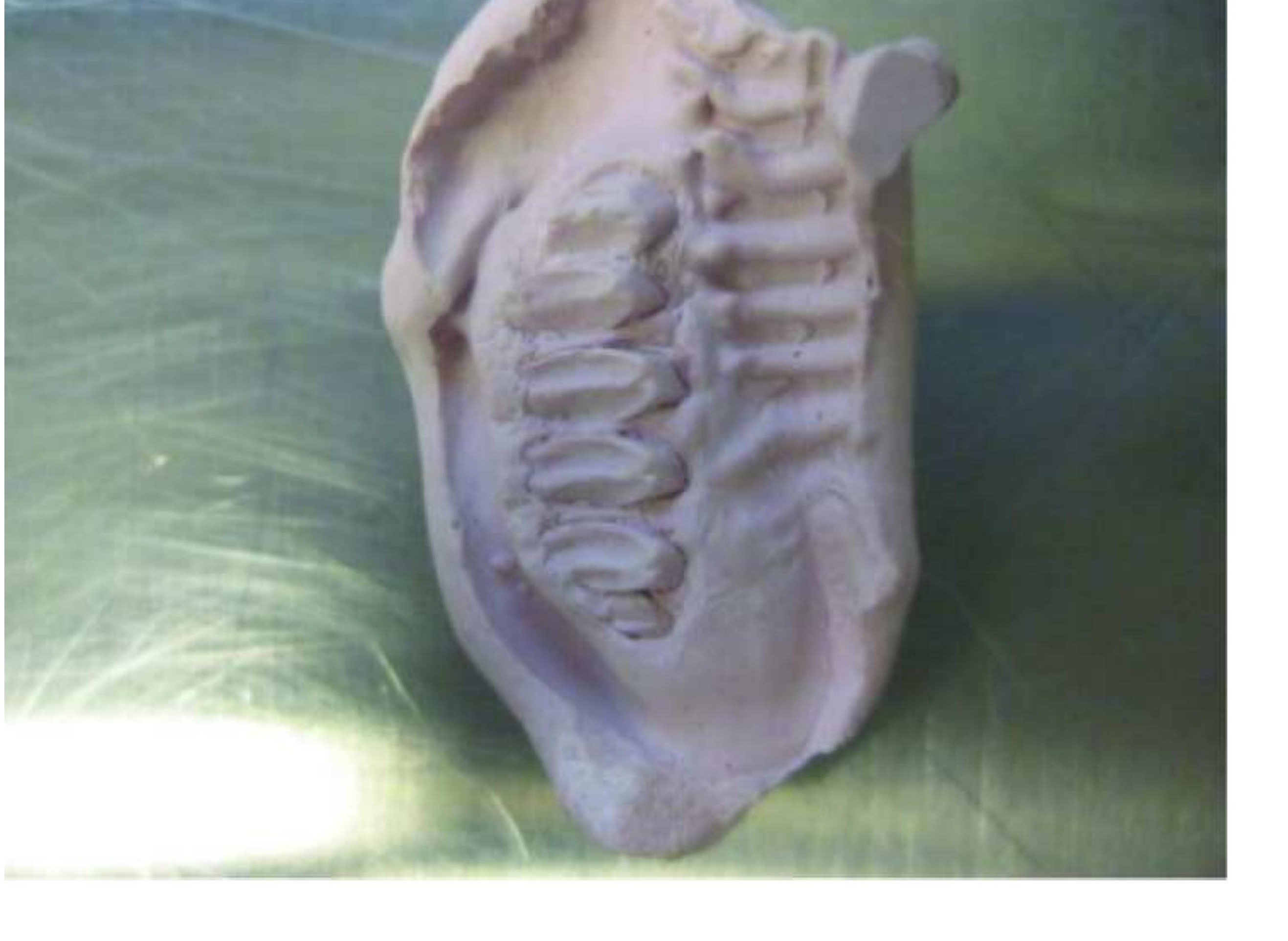


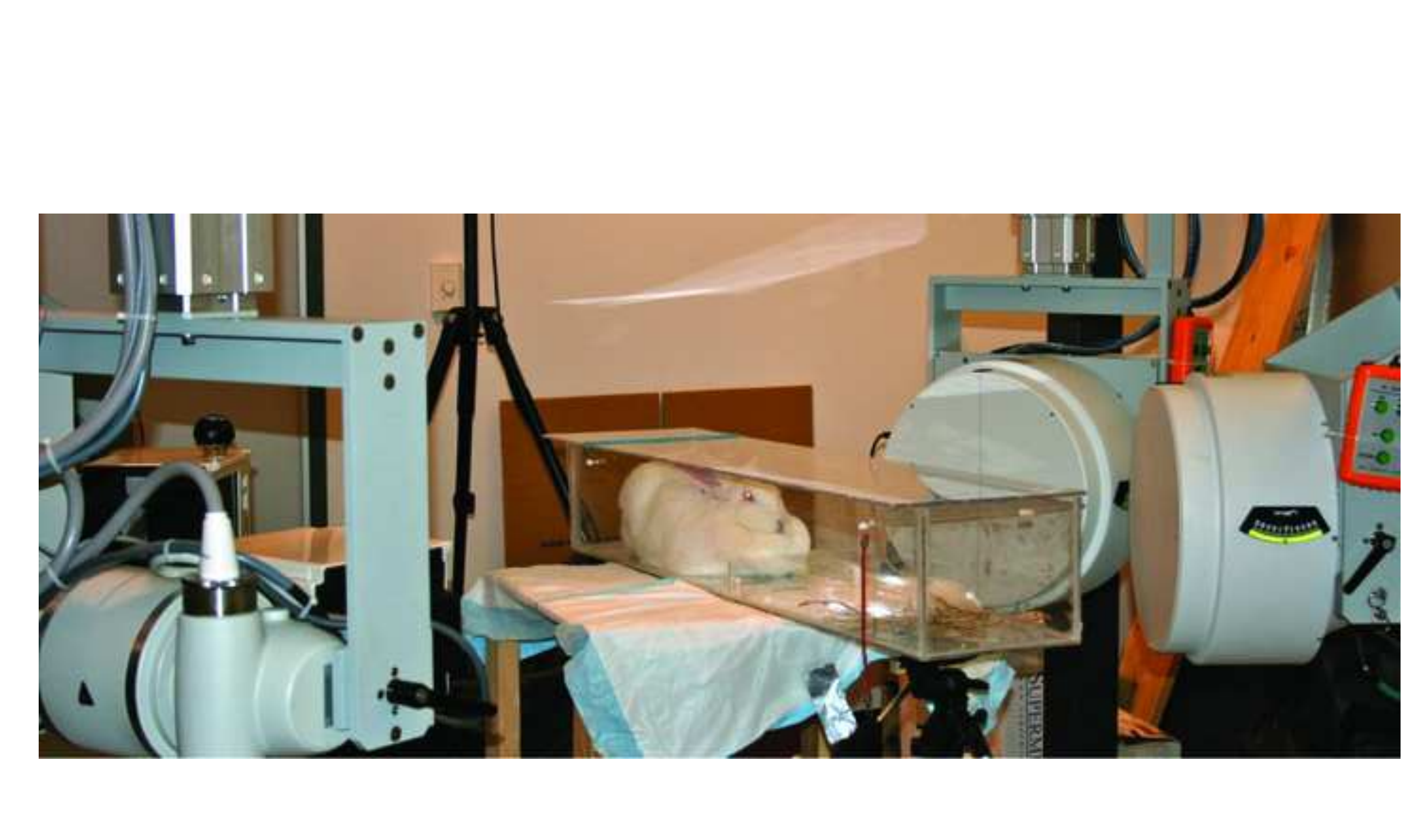

列

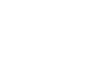

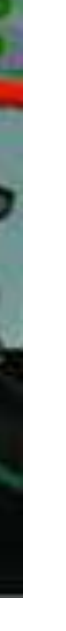

-
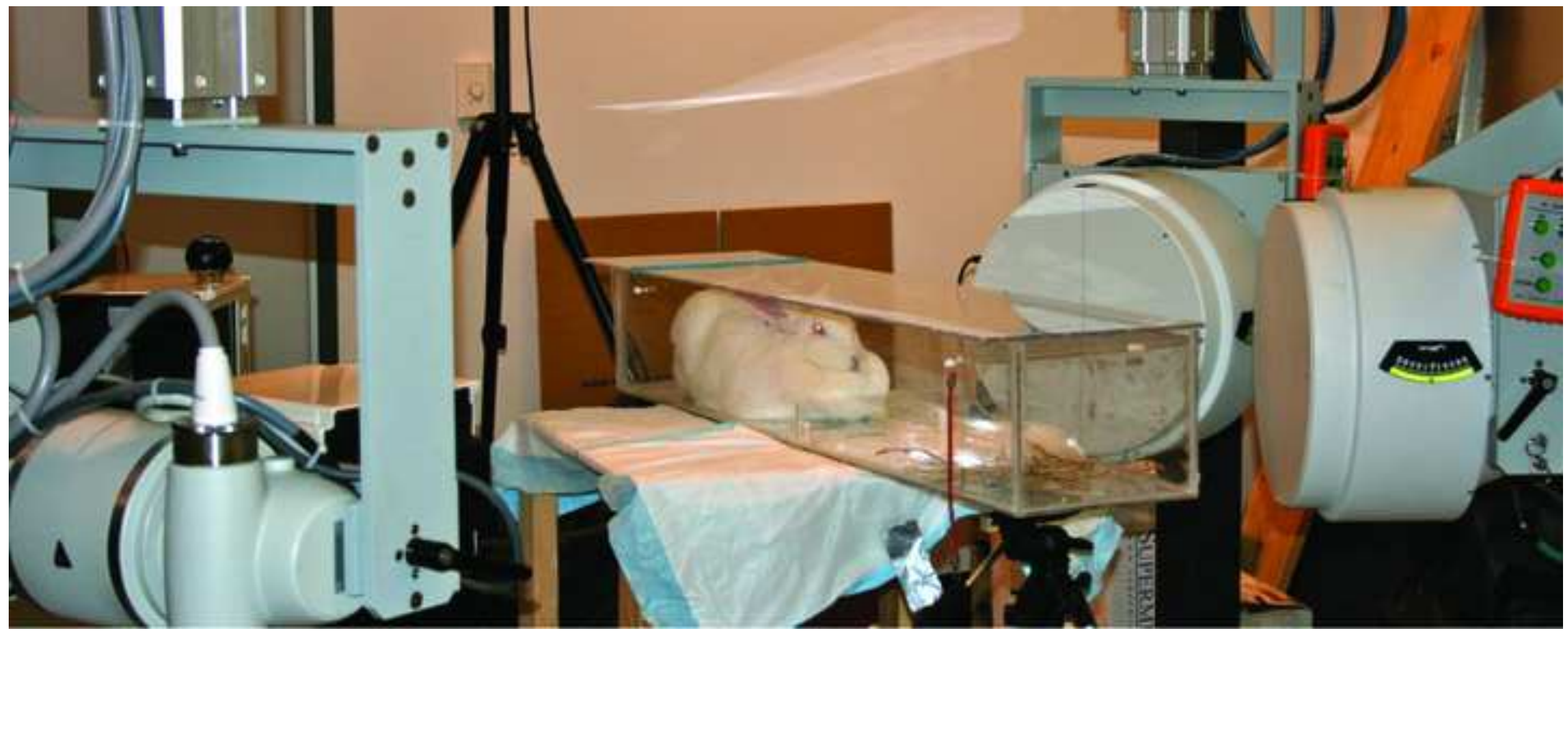
Figure2B

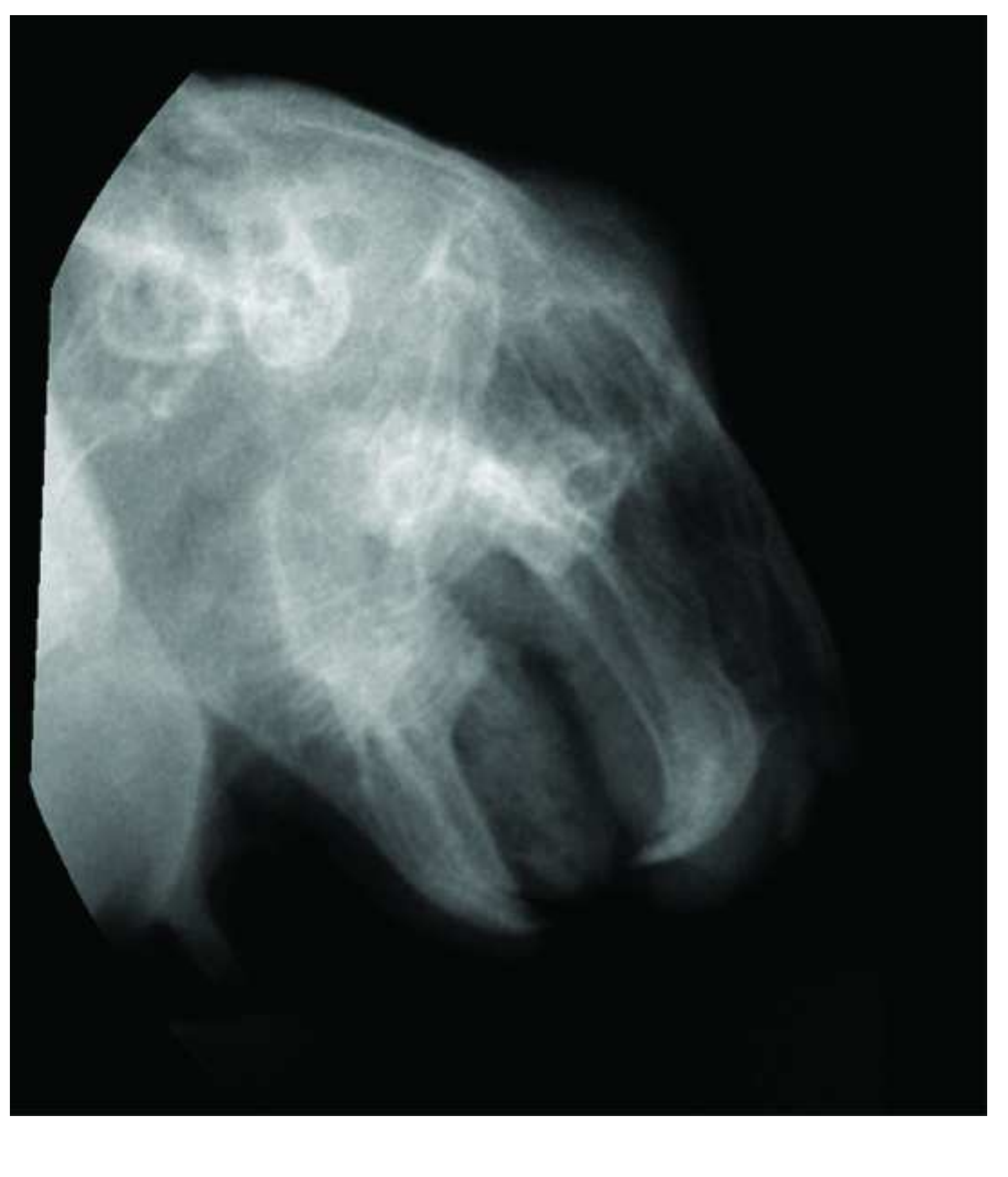

Figure2B

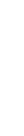

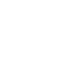

.

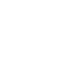

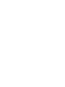
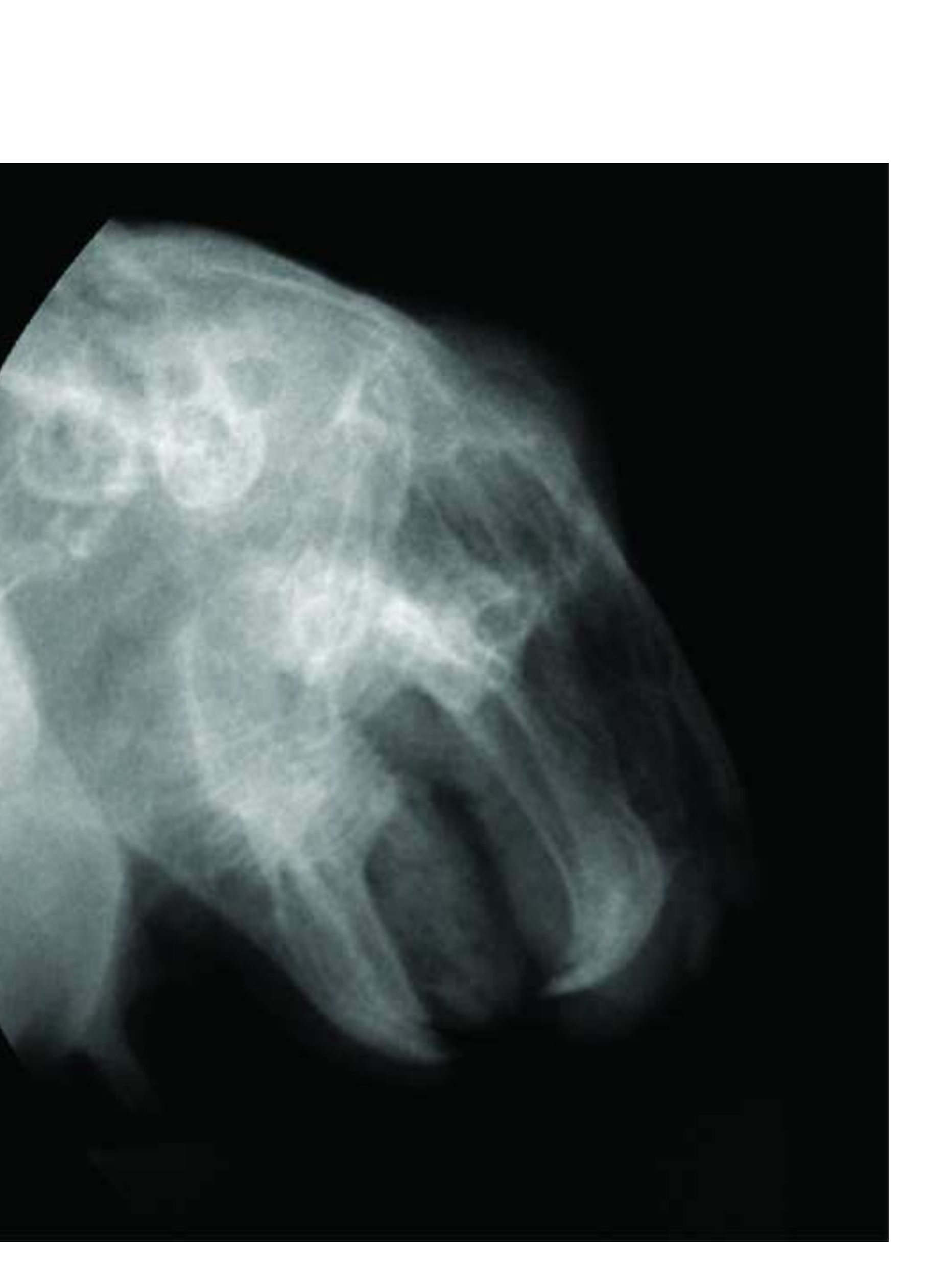


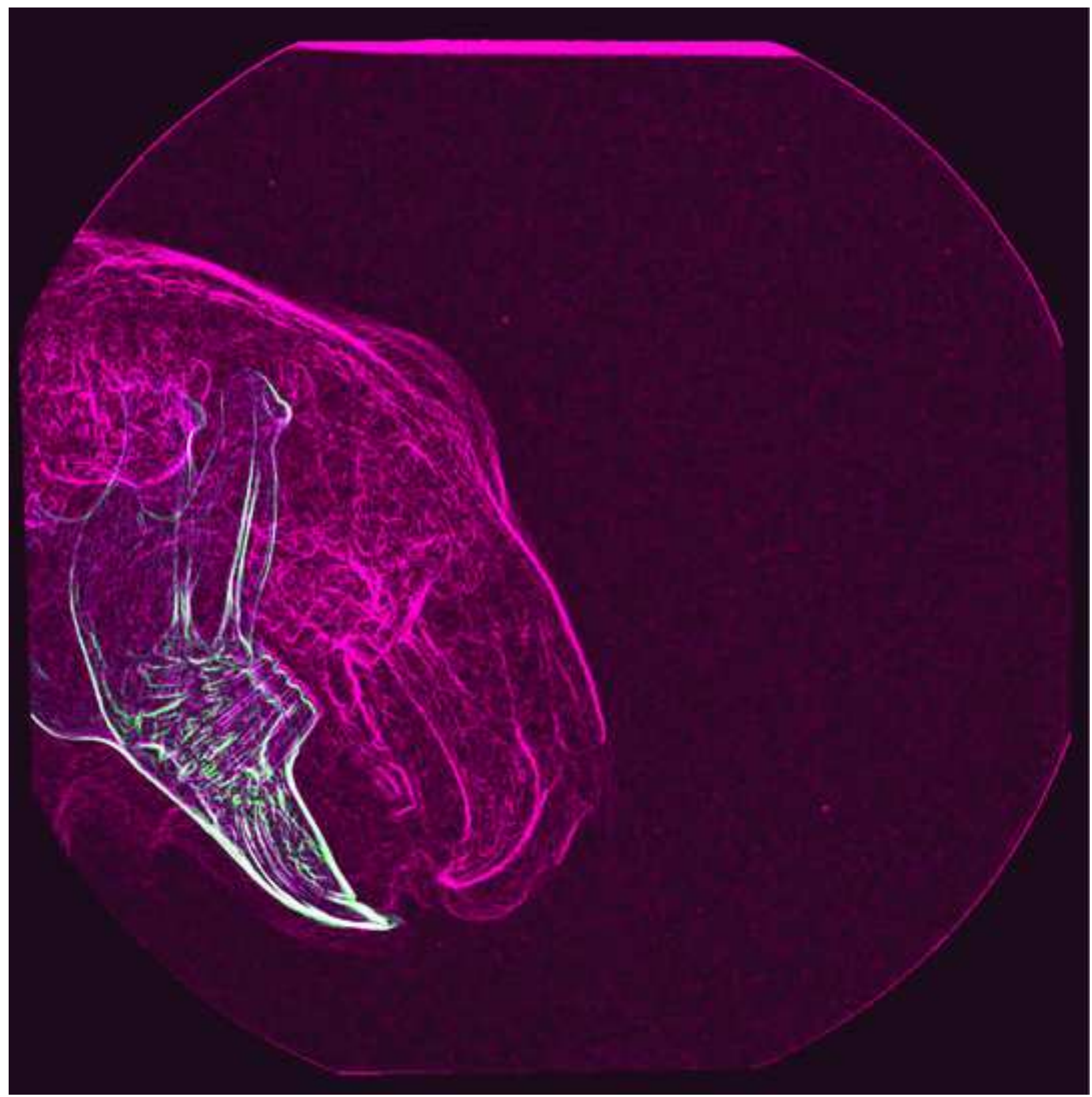




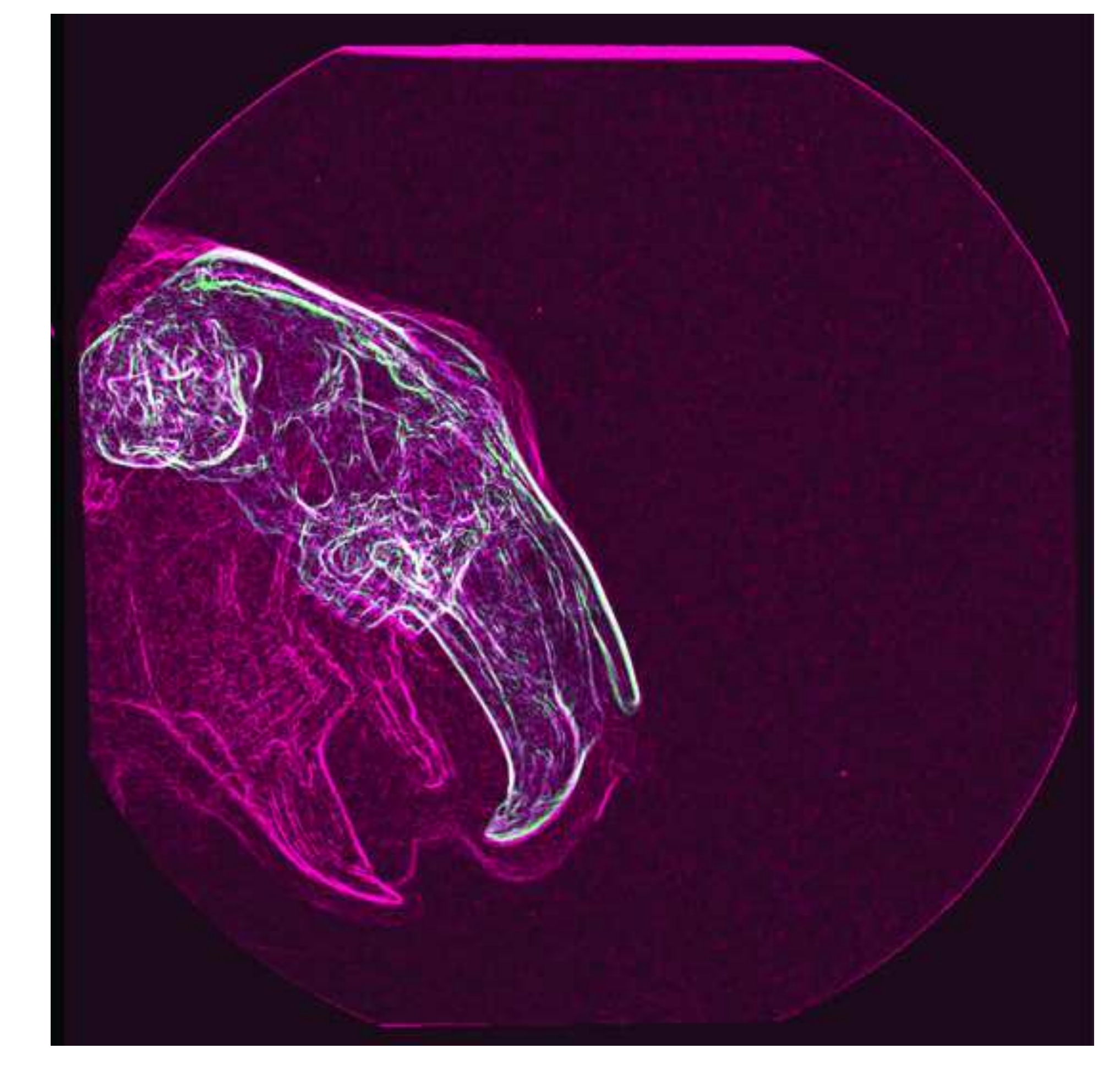

.

tr

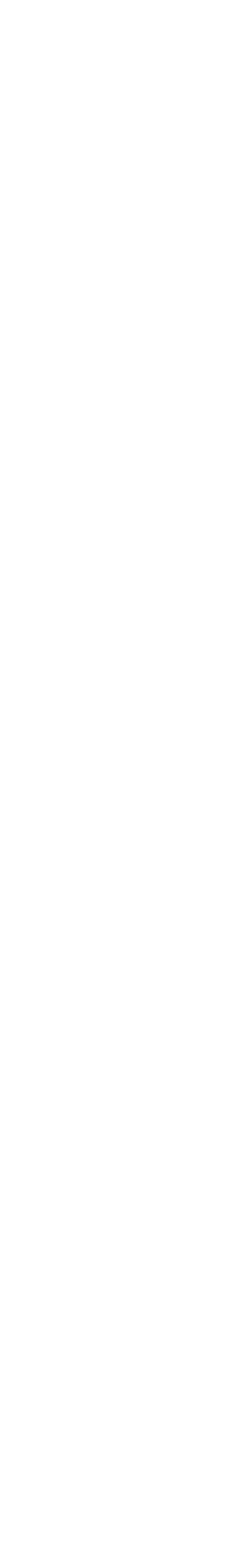
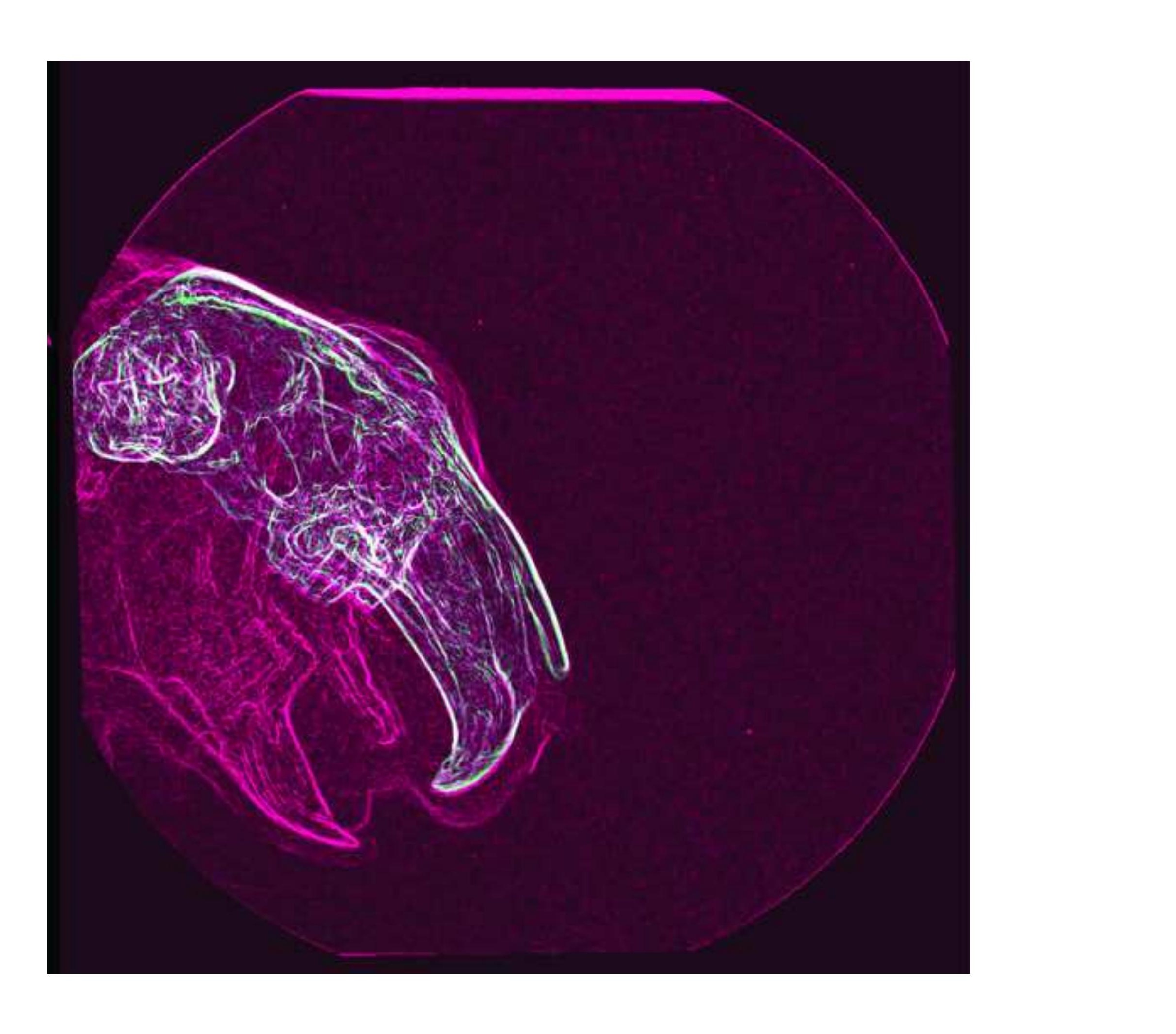


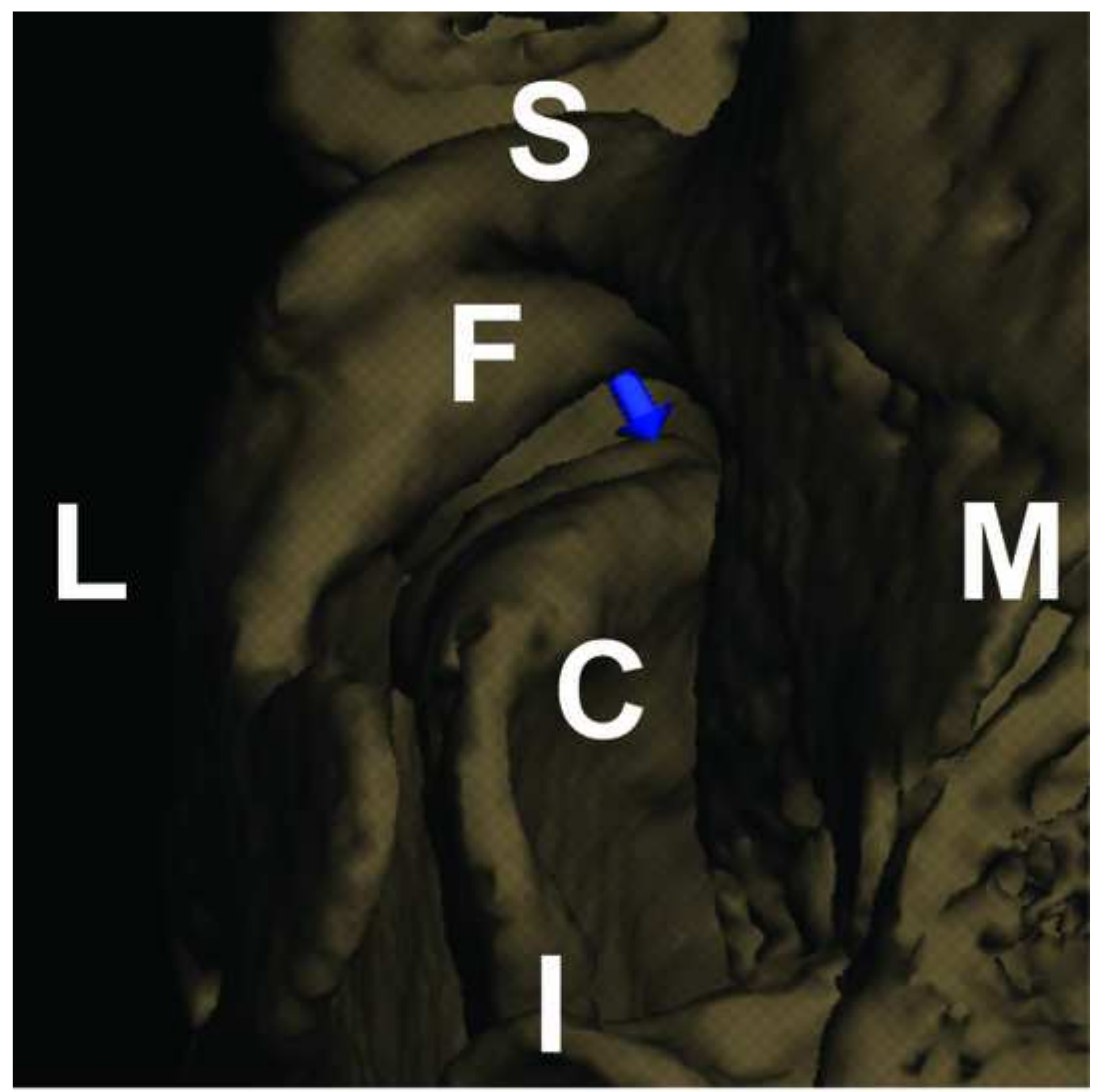


MW 


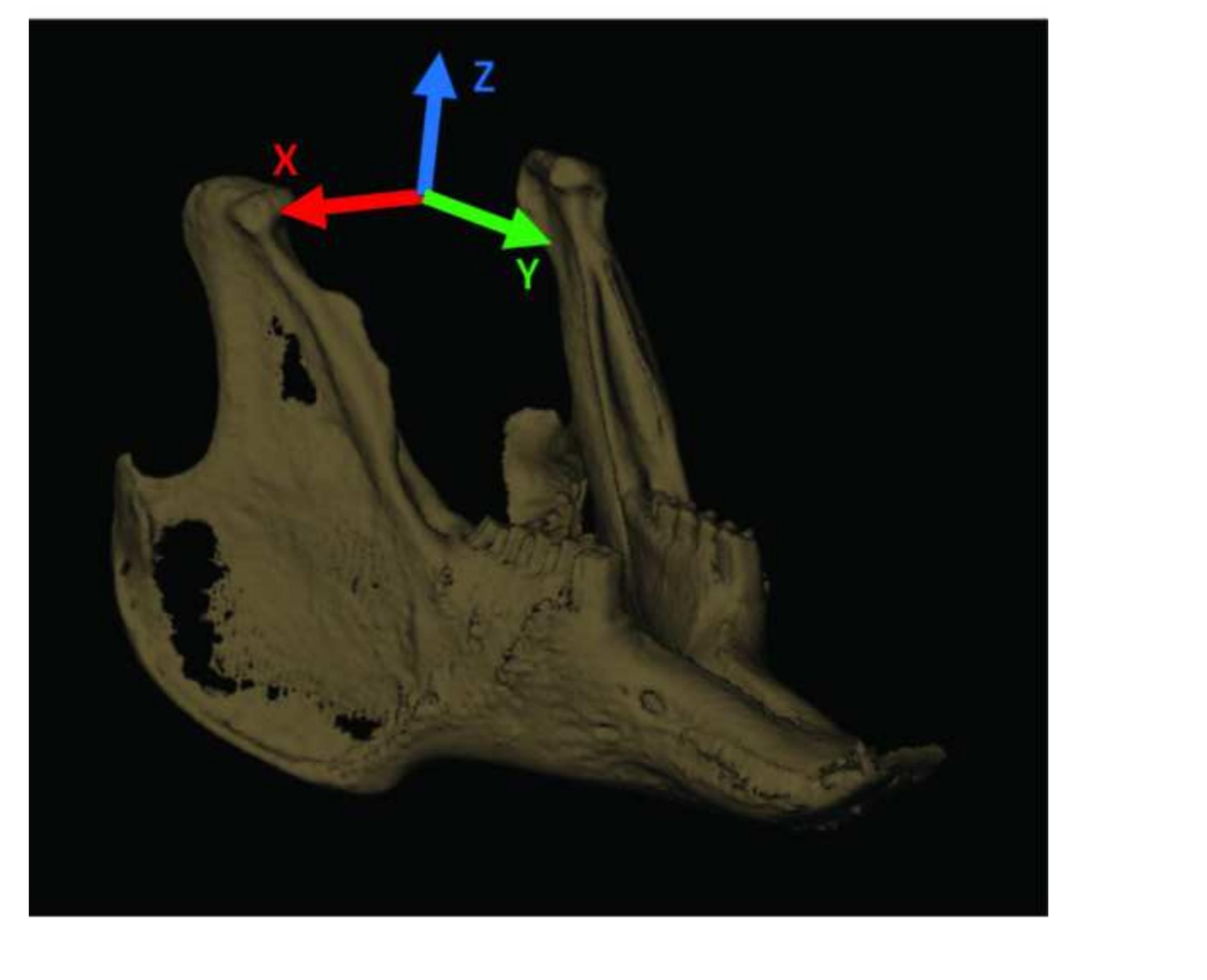

Figure3A
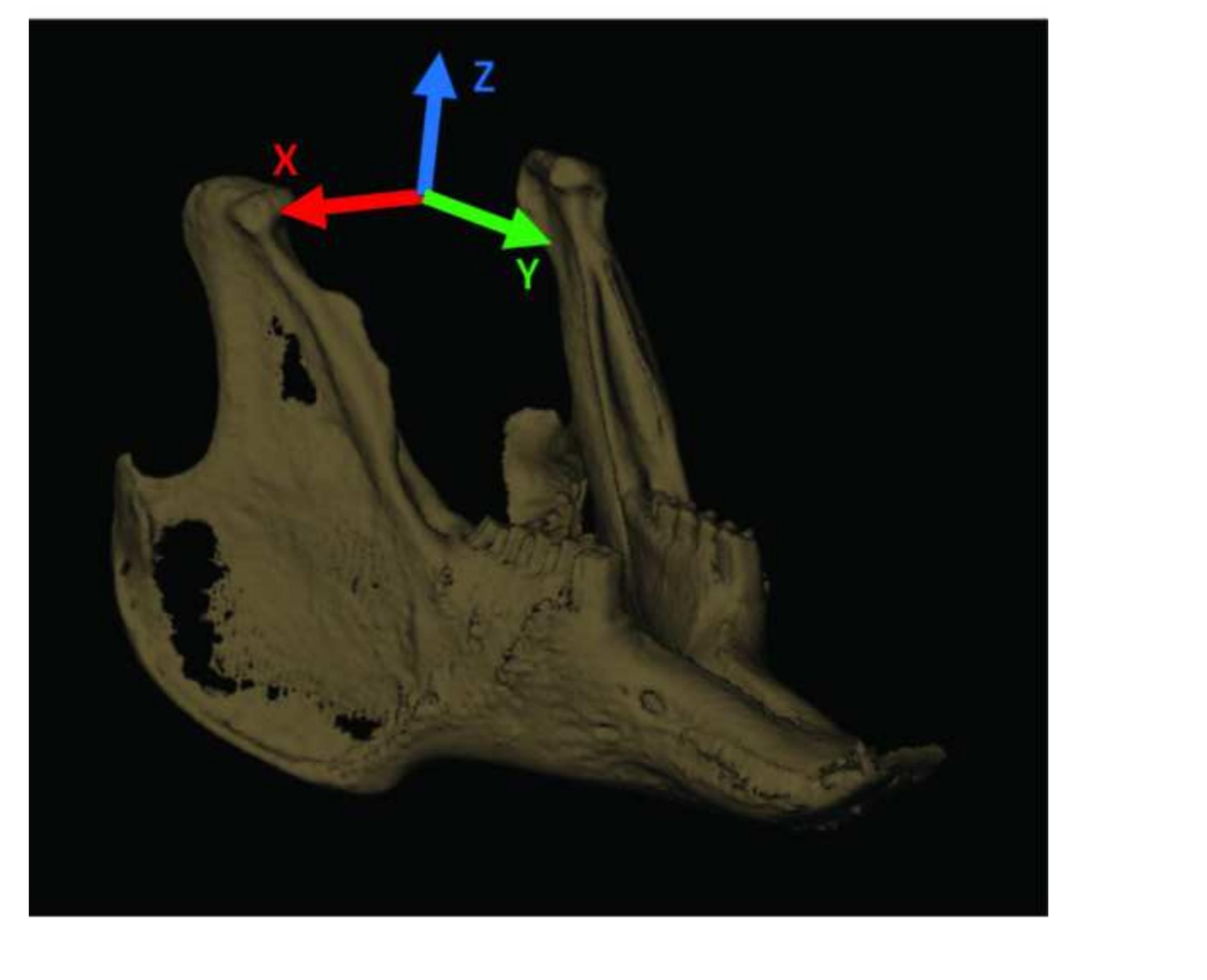

. \\ .
}
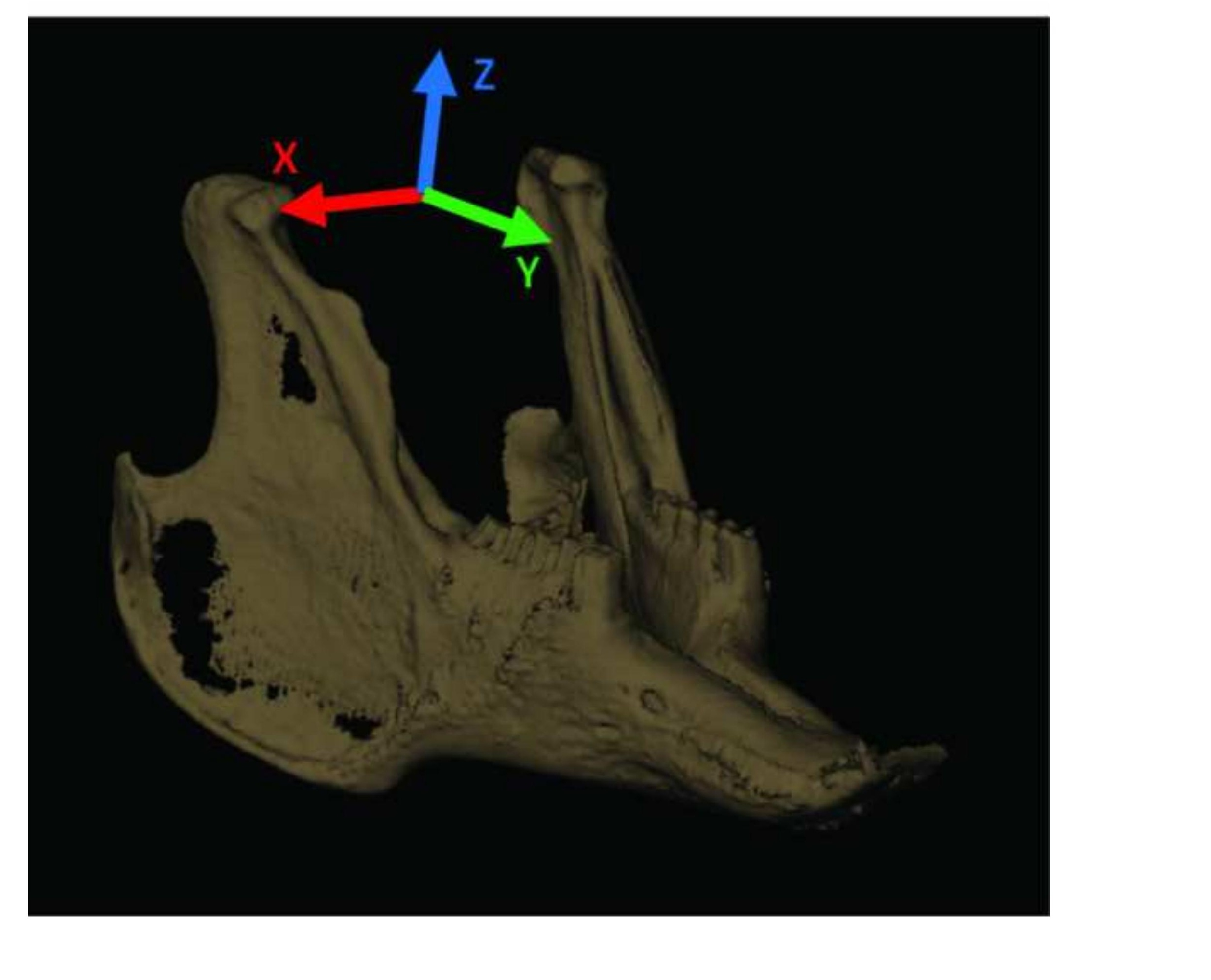
A

$\mathbf{R}$

\section{Le}
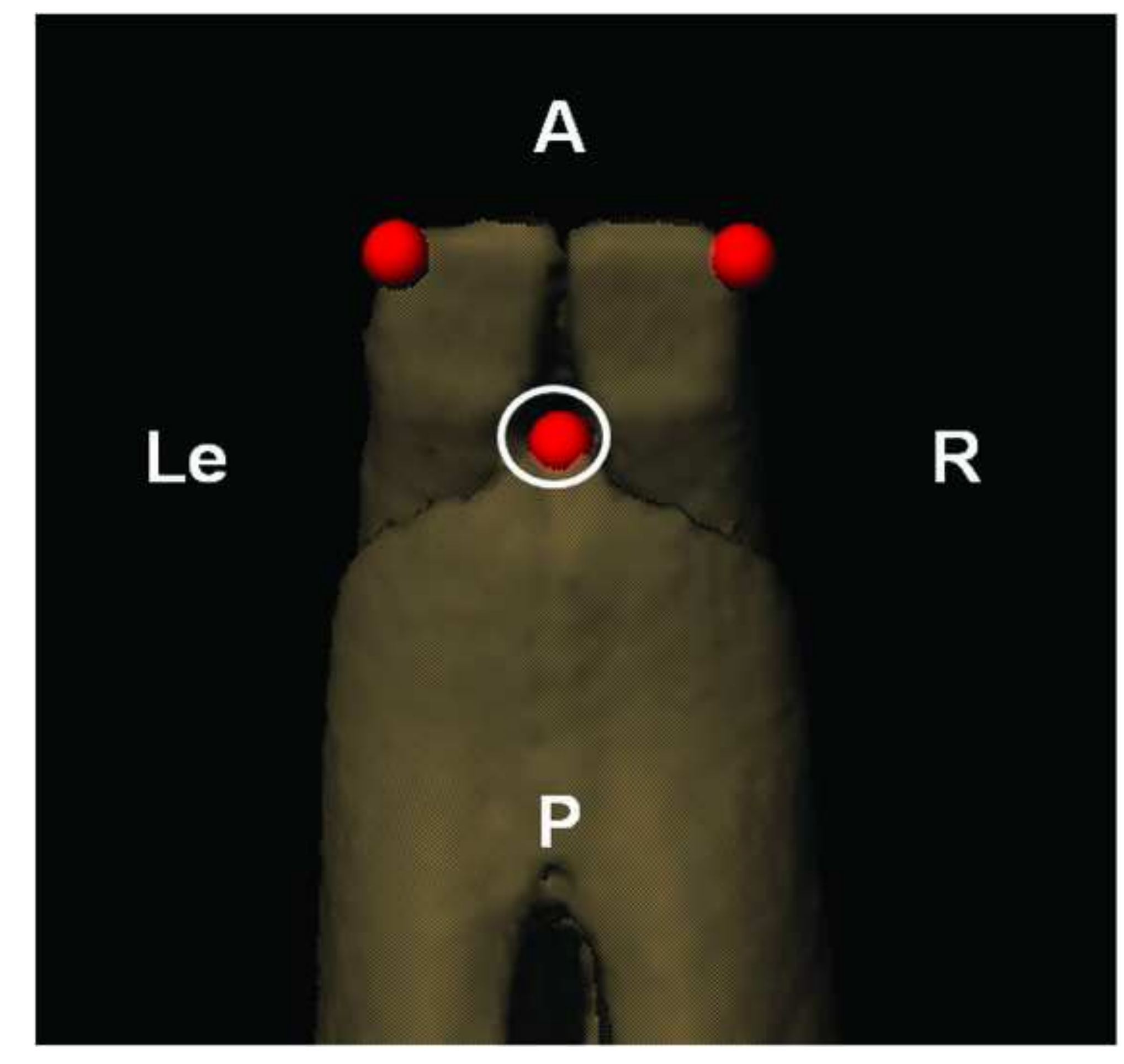

Figure 3 


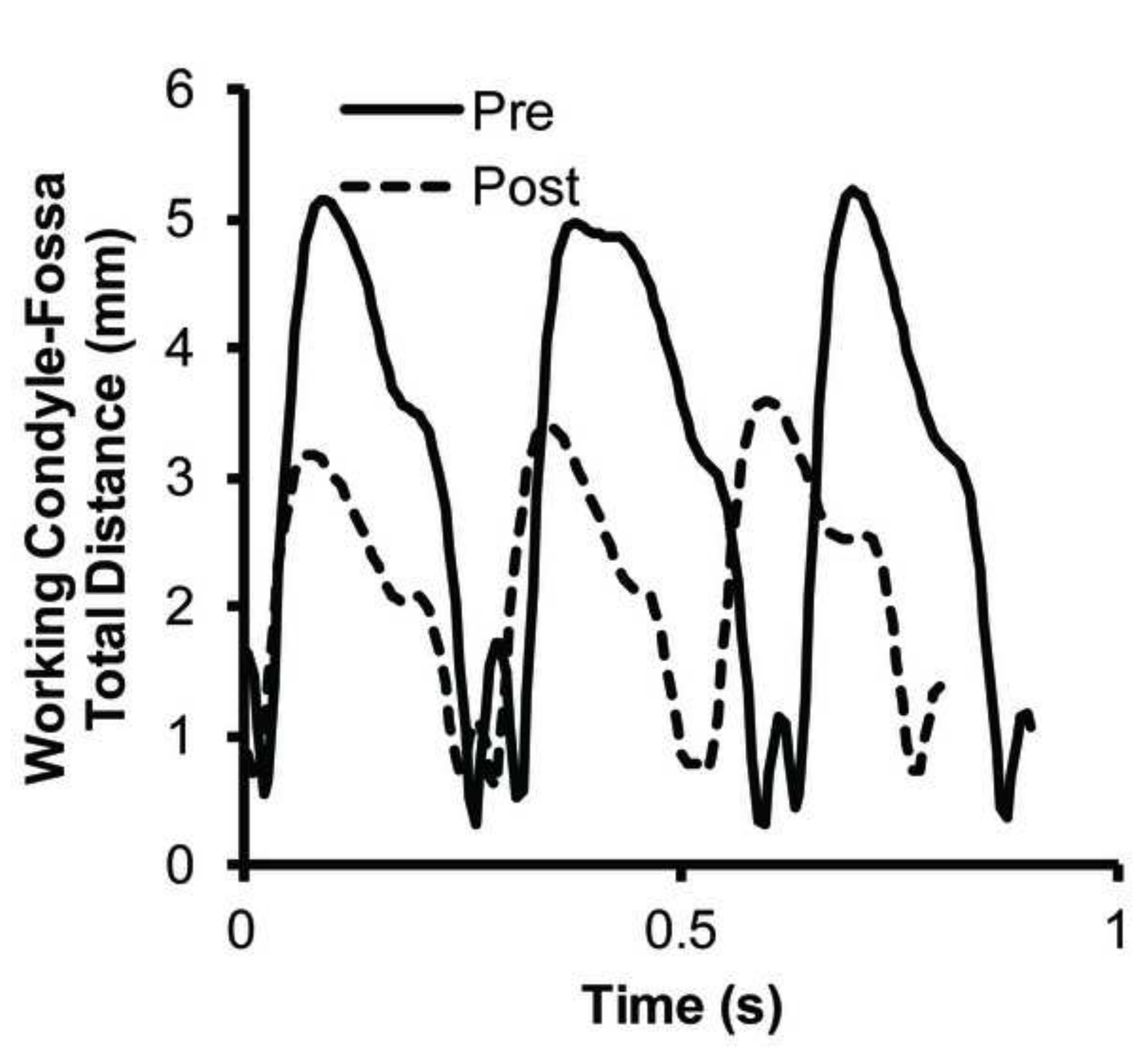




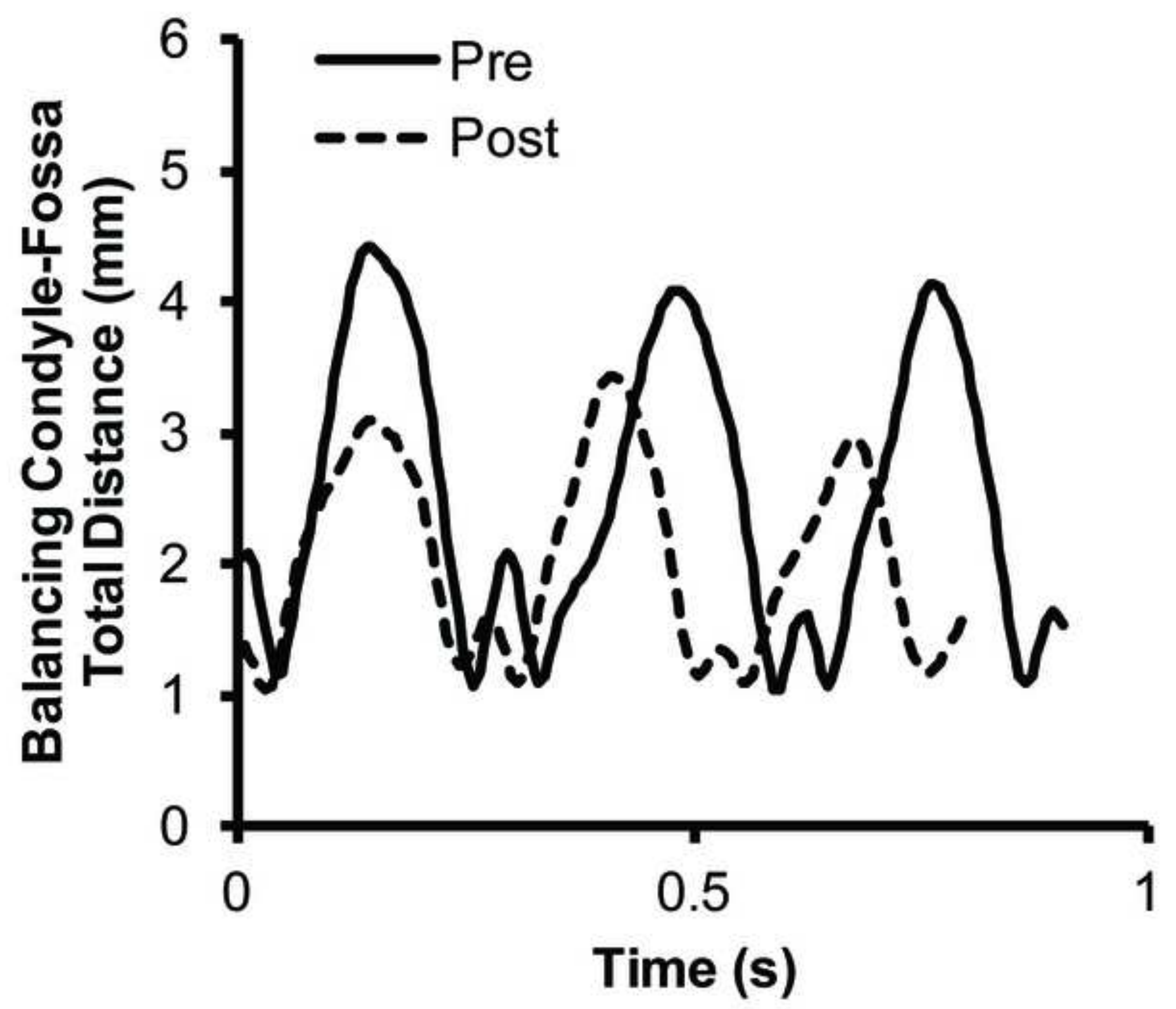




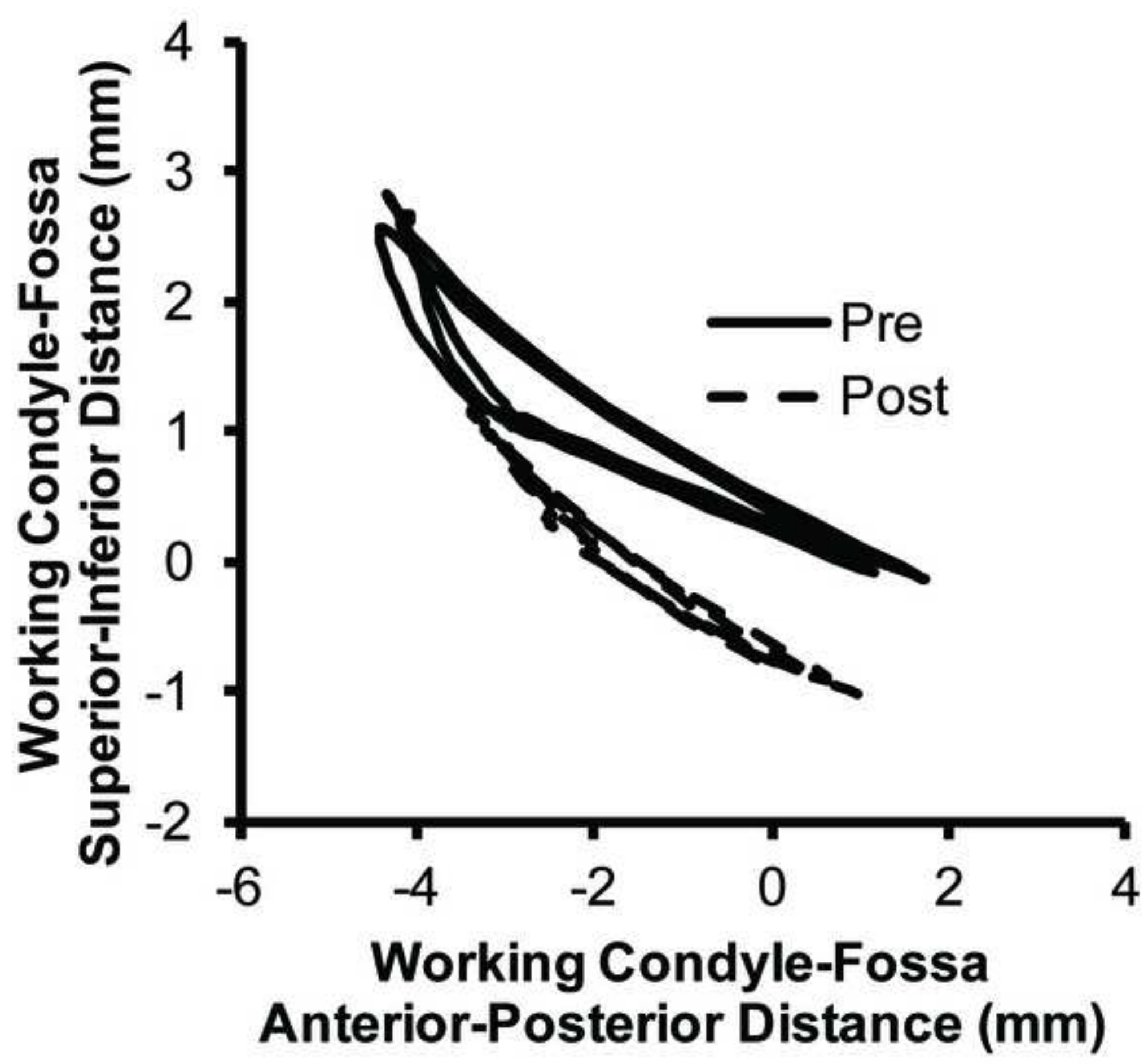




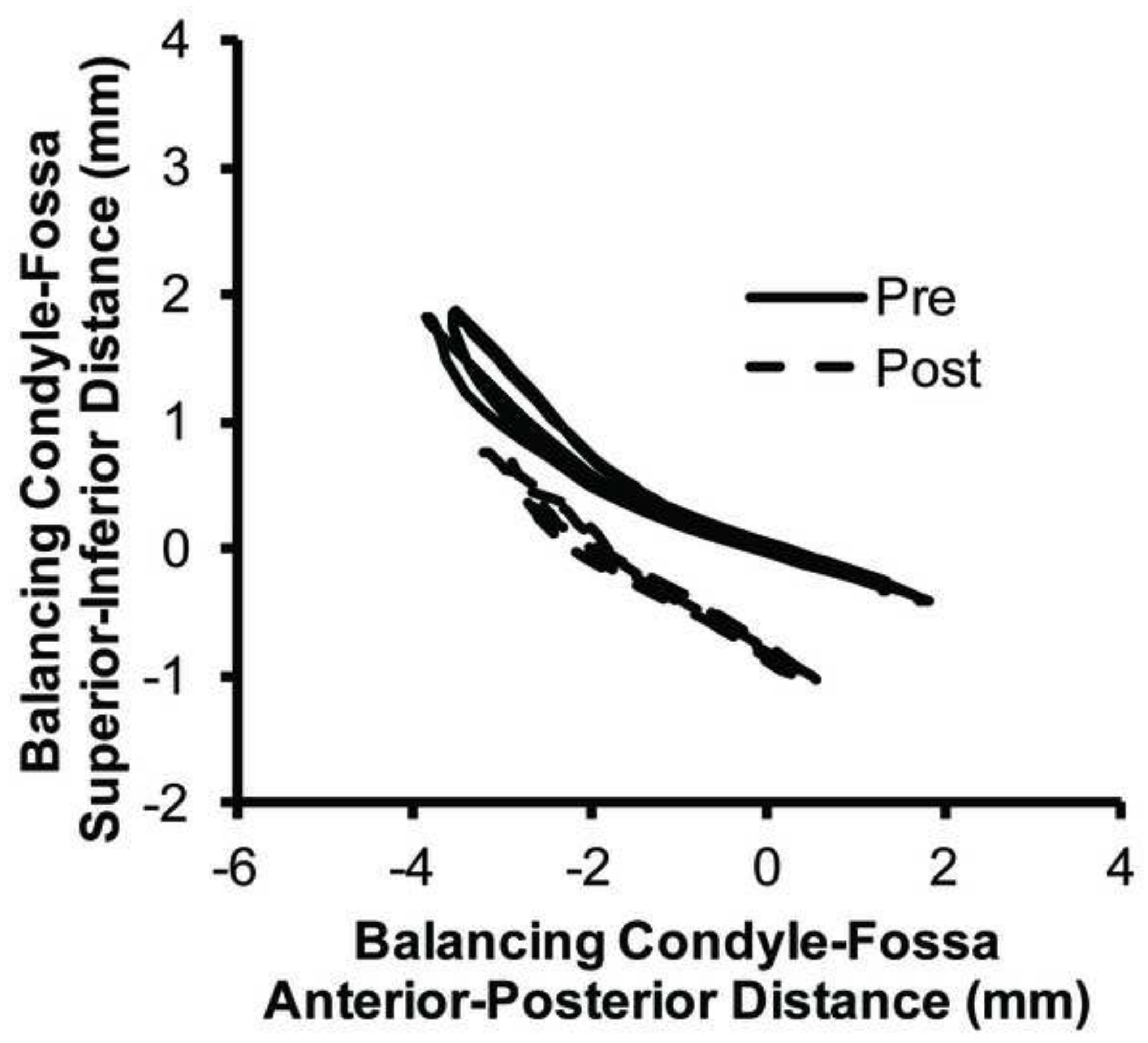




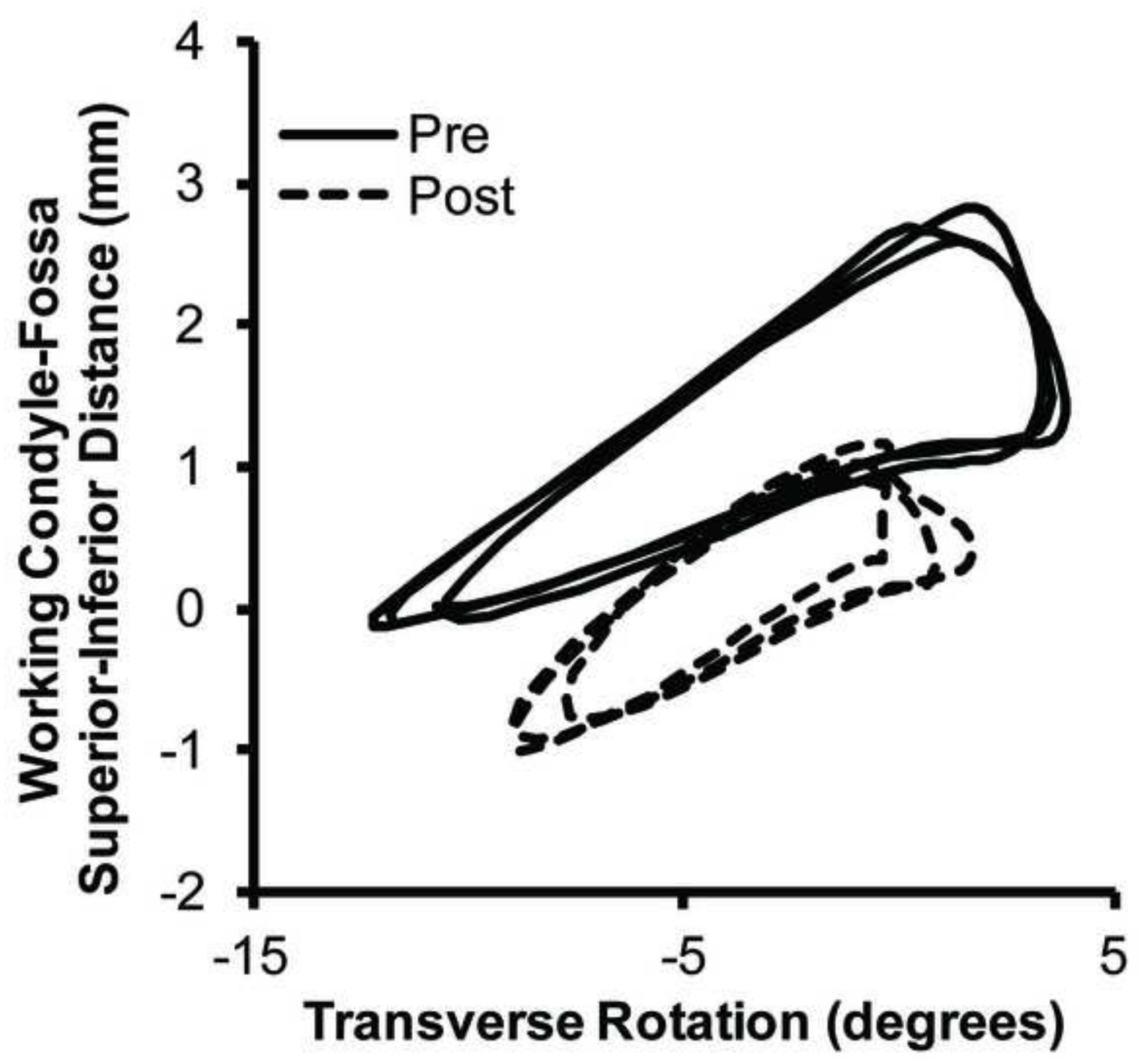




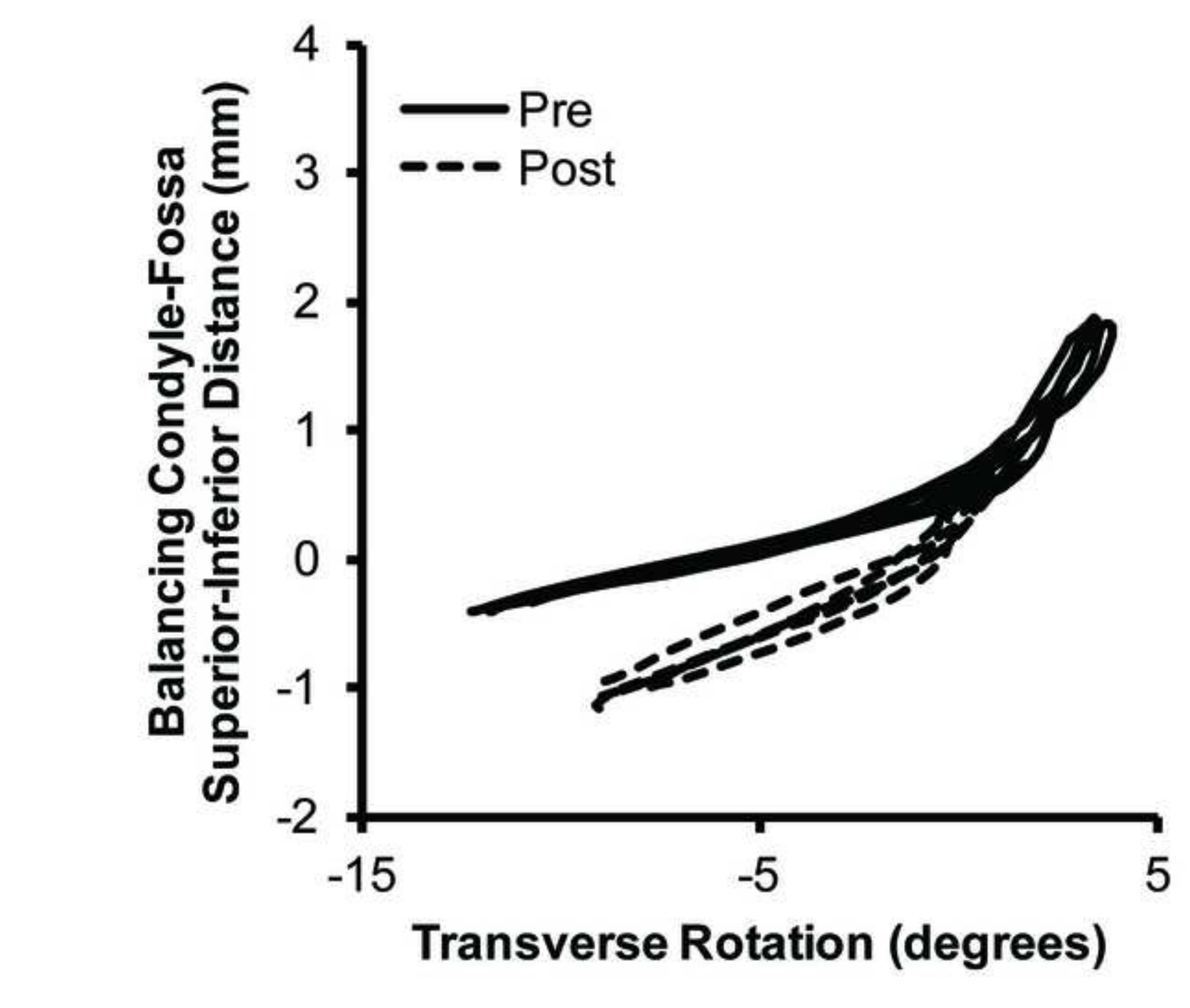

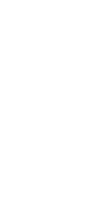

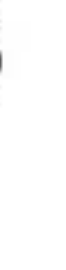

5

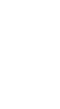

.




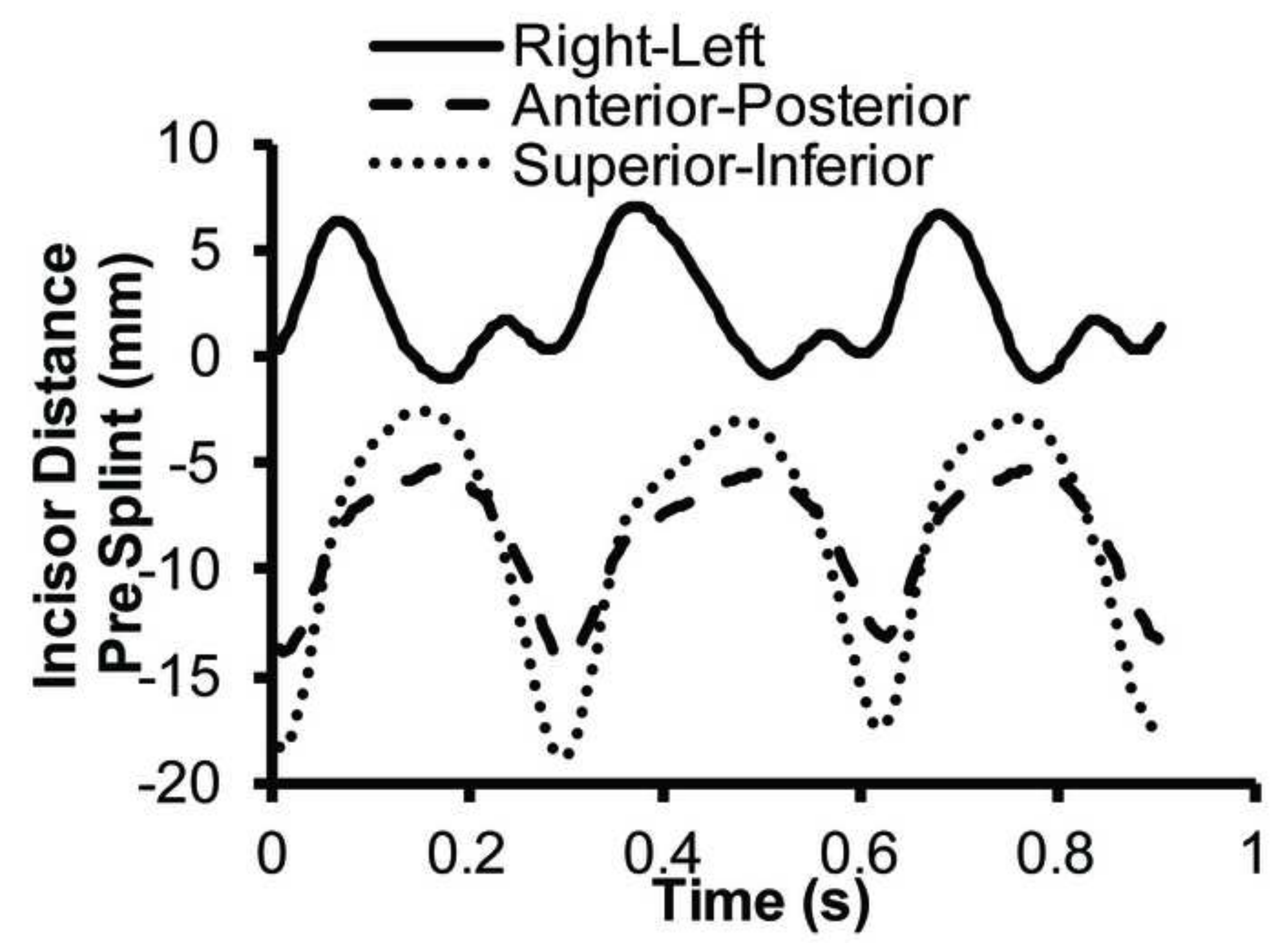




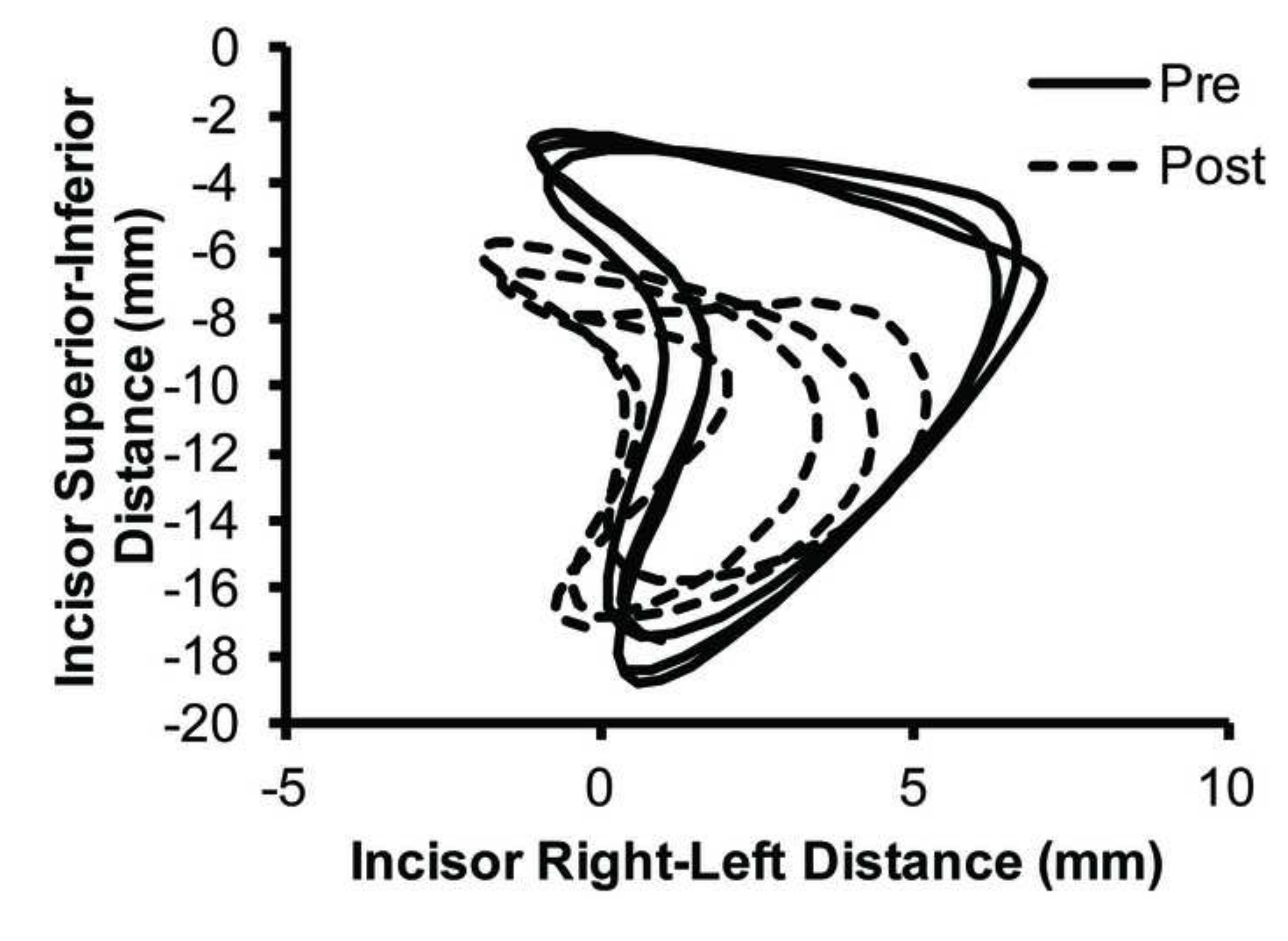

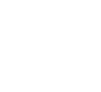

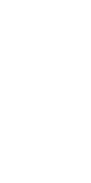

.

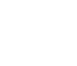

